\title{
Evidencias ambientales de cambios de nivel de la costa del Pacífico de México: terremotos y tsunamis ${ }^{1}$
}

\author{
María Teresa Ramírez-Herrera²
}

\begin{abstract}
RESUMEN
El estudio de sismos y tsunamis prehistóricos usando evidencias ambientales de cambios de nivel de la costa, donde se conservan los cambios abruptos de ambientes terrestres a marinos y viceversa, y los depósitos de tsunamis han proporcionado información valiosa en la evaluación del peligro por sismos y tsunamis. La costa del Pacífico mexicano es tectónicamente activa y ha experimentado numerosos sismos de magnitud grande $(M>7.5)$ en tiempos históricos, frecuentemente acompañados por cambios en el nivel de la costa y tsunamis ( $>50$ documentados desde 1732). Utilizando el estudio de caso de lagunas y marismas tropicales de la costa de Guerrero, mediante el enfoque proxy que integra geomorfología, estratigrafía, geoquímica, microfósiles y datación radiométrica, se muestra la posibilidad de documentar cambios de nivel de la costa inducidos tectónicamente. El método utilizado confirma evidencias de cambios ambientales que demuestran un probable terremoto acompañado de subsidencia de la costa y de un tsunami a los ca. 3400-3500 A.P. En general, la mayoría de los cambios relativos de nivel del mar observados en el Holoceno obscurecen cualquier señal climática durante este periodo en la costa mexicana del Pacífico.
\end{abstract}

Palabras clave: Cambios ambientales, marismas lagunares, sismo, tsunami, Holoceno.

\begin{abstract}
The study of prehistoric earthquakes and tsunamis using environmental evidence of coastal level changes, where sudden changes from terrestrial to marine environments, and vice versa, and where tsunami deposits are preserved, has provided valuable information in the earthquake and tsunami hazard assessment. The Pacific coast of Mexico is tectonically active and has experienced numerous large magnitude earthquakes $(M>7.5)$ in historical times, frequently accompanied by coastal-level changes and tsunamis (> 50 tsunamis recorded since 1732). Using the case study of tropical lagoons and marshes on the Guerrero coast, it is shown the feasibility of recording tectonically induced coastal level changes through the proxy method that integrates geomorphic, stratigraphic, geochemical, microfossil, and radiometric data. The applied method confirms evidence for environmental changes indicative of coastal subsidence and a potential great earthquake and tsunami by ca. 3400-3500 yr BP. In general, most of the Holocene relative sea-level changes observed obscure any climatic signal in this period on the Mexican Pacific coast.
\end{abstract}

Key words: Environmental changes, lagoon, marsh, earthquake, tsunami, Holocene.

1 Este estudio ha sido financiado por la UNAMPAPIIT IN123609 "Paleosismicidad de la Zona de Subducción Mexicana-buscando candidatos de grandes sismos y tsunamis", CONACYT-Repatriación. Artículo recibido el 3 de mayo de 2010, acep- tado el 24 de mayo de 2011 y corregido el 20 de junio de 2011.

2 Centro de Investigaciones en Geografía Ambiental, Universidad Nacional Autónoma de México (México). E-mail: mtramirez@ciga.unam.mx 
Las evidencias de cambios ambientales abruptos han sido ampliamente utilizadas para determinar cambios de nivel de la costa asociados con terremotos (Atwater \& Hemphill-Haley 1997; Hutchinson et al., 1997; Shennan et al., 1999; Cundy et al., 2000; Sawai, 2001; Chagué-Goff et al., 2002; Jankaew et al., 2008; Sawai et al., 2004; Sawai et al., 2008). El terremoto en el océano Índico en el 2004 mostró los cambios dramáticos que ocurrieron en el nivel de la costa, subsidencia y levantamiento en el rango de varios metros, que alteraron el curso natural de los ambientes costeros (Briggs \& Meltzner, 2005; Tobita \& Suito, 2006; Chini \& Bignami, 2008). Antes de 2004 el acuerdo convencional era que los grandes terremotos (magnitud $\geq 9$ ) podían producirse solamente donde las placas tectónicas son jóvenes, y donde el material flotante de la placa oceánica subduce bajo el margen continental con tasas de velocidad relativamente altas (Ruff \& Kanamori, 1980). El terremoto de Sumatra de 2004 cambió esta relación. Dicho terremoto demostró que la relación empírica entre la magnitud del sismo, la edad de la placa y la velocidad de convergencia de las placas, desarrollada por Ruff \& Kanamori (1980), debía ser reevaluada. Los recientes intentos realizados para determinar los diferentes factores que gobiernan la sismicidad y las variaciones de la zona de falla de la zona de subducción de Sunda-Andaman han producido resultados interesantes (Stein \& Okal, 2005; Ni et al., 2005; Henstock et al., 2006; Gervemeyer \& Tiwari, 2006; Subarya et al., 2006; Bilek, 2007). A pesar de estos valiosos estudios, y dado el corto tiempo de observaciones (el último siglo) sobre la distribución de la sismicidad en zonas de subducción, las correlaciones resultan de un periodo de muestra muy corto. La historia de grandes sismos es muy incompleta, dificultando los esfuerzos para entender dónde pueden ocurrir grandes sismos (Lomnitz, 1970; Ambraseys et al., 1994; Ambraseys et al., 2002; Farreras \& Sánchez, 1991; Farreras, 1997; Cisternas, 2005; Satake \& Atwater, 2007). Un análisis extenso sobre la recurrencia de grandes sismos requiere más información que la actualmente disponible, basada solamente en mediciones instrumentales y del registro histórico (Hutchinson et al., 1997; Williams \& Hutchinson, 2000; Kelsey et al., 2005; Satake \& Atwater, 2007). Específica- mente se requiere más información sobre el registro a largo plazo de los sismos (miles de años) solo disponible a través de estudios de paleosismología, conjuntamente con la historia escrita de los sismos y tsunamis (Kawana \& Pirazzoli, 1985; Atwater, 1987; Atwater \& Moore, 1992; Berryman, 1993a; Berryman, 1993b; Minoura et al., 1994; Minoura et al., 1996; Atwater et al., 1995; Atwater \& Hemphill-Haley, 1997, 2004; Chappell et al., 1996; Otta \& Chappell, 1996; Satake et al., 1996; Clague et al., 2000; Chagué-Goff \& Goff, 1999; Sawai, 2001; Sawai et al., 2004; Nanayama et al., 2003; Audemard, 2005; Nelson et al., 1996; Nelson \& Personious, 1996; Cisternas, 2005; Cisternas et al., 2005; Natawidjaja et al., 2006; Satake \& Atwater, 2007; Jankaew et al., 2008; Monecke et al., 2008). Los grandes sismos producen efectos cosísmicos tan claros y extensos en los ambientes naturales costeros que su expresión acumulativa se puede identificar en el campo una vez transcurrido un intervalo de tiempo geológico (Nelson et al., 1996; Michetti et al., 2005).

Es relativamente escasa la información disponible sobre paleosismicidad de límites de placa de subducción complejos (Nanayama et al., 2003; Atwater et al., 2004; Sawai et al., 2004; Cisternas et al., 2005; Natawidjaja et al., 2006; Satake \& Atwater, 2007) como la zona de subducción mexicana, caracterizada por cambios en las tasas de convergencia de las placas, su ángulo de subducción, la edad del piso oceánico y la subducción de grandes fracturas y dorsales. Algunas zonas de subducción con mayor información de paleosismos sirven como tipos análogos o contrastantes de fallamiento de megathrust (megafalla inversa), como la subducción de Cascadia (Atwater \& Moore, 1992; Atwater et al., 1995; Atwater et al., 1997; Hutchinson et al., 1997; Williams \& Hutchinson, 2000), la de Nazca en Chile (Cisternas et al., 2005), la de Sunda-Andaman y la de Japón (Nanayama et al., 2003; Atwater et al., 2004; Sawai et al., 2008). 


\section{La zona de subducción mexicana-sismotectónica, tsunamis y tormentas}

\section{La zona de subducción mexicana (ZSM)}

En el sur de México la subducción de la placa Rivera-Cocos bajo la placa norteamericana se extiende en una distancia de $>1.500$ $\mathrm{km}$ (Figura $\mathrm{N}^{\circ} 1$ ). La convergencia de placas en la ZSM presenta tasas de velocidad que varían de 2,0 a 5,0 cm/año en el límite de placas Rivera-Norteamérica, y de 5,2 a 6,8 cm/año en el límite de placas Cocos-Norteamérica (Figura No 1) (DeMets et al., 1994). El ángulo de la subducción es subhorizontal al oeste de $\sim 96^{\circ} \mathrm{W}$, con un ángulo inicial $>15^{\circ}$ que se inclina $>30^{\circ}$ cerca de la costa y subsecuentemente se vuelve subhorizontal. El ángulo de subducción aumenta a $\sim 45^{\circ}$ al este de los $\sim 96^{\circ} \mathrm{W}, \mathrm{y}$ al noroeste de Guerrero $\left(\sim 102^{\circ} \mathrm{W}\right)$ la placa subduce con ángulo $>30^{\circ}$ a profundidades mayores de $40 \mathrm{~km}$. Bajo la zona sismogénica de la microplaca de Rivera el ángulo es de $\sim 10^{\circ}$ a unos $20 \mathrm{~km}$ de profundidad, incrementándose a $46^{\circ}$ a una profundidad de 65 km (Pardo \& Suárez, 1995).

Figura $\mathrm{N}^{\circ} 1$

Sismicidad y tectónica de la costa del Pacífico en Guerrero, México. MAT-Trinchera Mesoamericana

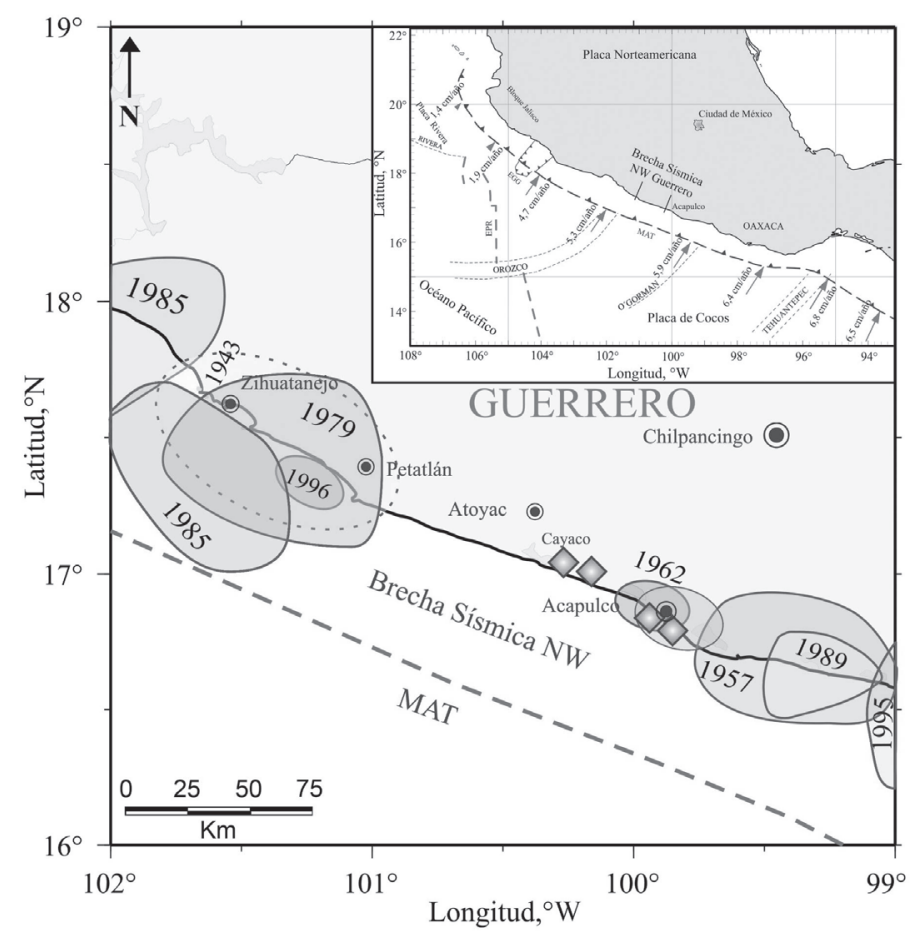

Símbolos: Los círculos sombreados muestran áreas de ruptura y los números indican los años de los eventos sísmicos de subducción más importantes durante el siglo pasado. Las tasas de convergencia de placas se muestran en cm/año, mientras que las flechas indican la dirección de convergencia. Los rombos enseñan el sitio de estaciones GPS y de nivelación. El recuadro muestra el marco tectónico del área de estudio: Rivera, Orozco, O'Gorman y Tehuantepec-zonas de fracturas, EGG-graben El Gordo, EPR-Dorsal del Pacífico este. El área aproximada de la Figura N N 2 se muestra entre líneas marcadas como brecha sísmica noroeste de Guerrero. Fuente: Elaboración propia. 
La ZSM ha sido subdividida en varias regiones sismo y morfotectónicas (Pardo \& Suárez, 1995; Ramírez-Herrera \& UrrutiaFucugauchi, 1999; Ramírez-Herrera et al., 1999). Los sismos superficiales grandes que ocurren en la ZSM son generalmente eventos de falla inversa, y representan el desplazamiento de las placas de Cocos-Rivera y la cabalgante placa de Norteamérica (Pardo \& Suárez, 1995). Durante los dos últimos siglos han ocurrido sismos superficiales grandes $(\mathrm{Mw}>7)$ en la ZSM, en segmentos de 100-200 km de longitud, con intervalos de recurrencia de 30-60 años (Singh et al., 1981; Singh \& Suárez, 1986). El potencial de eventos sísmicos mayores $(M>8)$ se confirma con el gran sismo de Jalisco de 1932 (Ms $=8,1)$, el evento de Colima-Jalisco de $1995(M w=8,0)$ y el sismo de Michoacán de 1985 (Mw =8,1); este último devastó áreas densamente pobladas de la Ciudad de México (población de 20 millones). El sismo de Michoacán provocó 10.000 muertes, 300.000 damnificados, y cuyas pérdidas económicas fueron del rango de cientos de millones de dólares (UNAM Seismology Group, 1986). La máxima longitud de la ruptura en la ZSM en tiempos históricos se estima en ca. $200 \mathrm{~km}$, correspondiendo al evento de Jalisco en 1932 (Kostoglodov \& Ponce, 1994).

\section{Brechas sísmicas de la ZSM}

Varias brechas sísmicas fueron identificadas a lo largo de la ZSM por Singh et al. (1981). Desde entonces, sismos grandes han cerrado las brechas de Guerrero-Oaxaca (Ometepec Ms =6,9 y 7,0, 1982), $\mathrm{Mi}$ choacán (Ms $=8,1,1985)$ y Jalisco (Mw $=8,0$, 1995). Dos rasgos batimétricos del piso de la placa de Cocos, la fractura de Orozco al norte y la de O'Gorman al sur, se extienden casi perpendiculares a la trinchera, definiendo la zona sismogénica completa en Guerrero (Nishenko \& Singh, 1987) (Figura $N^{\circ}$ 1). La brecha sísmica de Guerrero, donde se espera que ocurra un sismo grande $\mathrm{Mw}$ 8,1-8,4, está subdividida en los segmentos noroeste y sureste (Suárez et al., 1990; Anderson \& Menking, 1994; Valdés-González \& Novelo-Casanova, 1998). El segmento noroeste aparentemente se rompió el 16 de diciembre de 1911 produciendo un sismo grande (Ms 7,8), y el segmento sureste se rompió el 28 de julio de 1957 y el 15 de abril de 1907, produciendo otros de escala igual de grandes (Ms 7,8 y 7,9 respectivamente) (Ortiz et al., 2000). Sin embargo, el segmento noroeste de la brecha de Guerrero, entre Acapulco y Petatlán, sobresale como la región con mayor potencial sísmico, porque se sabe que esta región ha sufrido sismos grandes destructivos pero no ha experimentado ninguno desde 1911 (Suárez et al., 1990; Anderson \& Menking, 1994; Valdés-González \& Novelo-Casanova, 1998; Ortiz et al., 2000).

Información histórica indica que un terremoto, de magnitud equivalente a 8,5 rompió aproximadamente $450 \mathrm{~km}$ de longitud de la ZSM, con un tsunami que inundó ca. $6,5 \mathrm{~km}$ tierra adentro, un fenómeno no observado durante el periodo de mediciones instrumentales (Núñez Cornú et al., 2008; Suárez, 2007). La ocurrencia de estos grandes eventos, como el que sucedió el 28 de marzo de 1787 en la costa de Oaxaca, lleva a preguntar si terremotos como este, o aún más grandes, pueden ocurrir en otros segmentos de la ZSM. Todavía quedan sin respuesta las preguntas: ¿la ZSM puede producir sismos de magnitud Mw =9 o mayor?; si las condiciones son adecuadas, ¿se puede producir un tsunami transoceánico? y ¿con qué frecuencia y regularidad ocurren?

\section{Tsunamis en la ZSM}

Típicamente, la relación de tsunamis en México proviene de registros de mareas, los que en México comenzaron en 1952. Los datos históricos e instrumentales indican que al menos 50 tsunamis han llegado a las costas del Pacífico de México desde 1732 (Farreras \& Sánchez, 1991). De estos tsunamis, treinta y cuatro se originaron por fuentes sísmicas locales (Farreras \& Sánchez, 1991). La máxima altura de ola que se ha registrado en los mareógrafos ha sido de $3 \mathrm{~m}$ para los más recientes. Sin embargo, los datos históricos de los tres últimos siglos sugieren que estas alturas pueden ser mayores. 


\section{Mareas y tormentas}

La costa de Guerrero, de acuerdo a la estación mareográfica de Acapulco, muestra valores de alturas de la marea astronómica relativamente bajos; así el nivel de pleamar media es de 0,236 $\mathrm{m}$ y el nivel de bajamar media es de $-0,238 \mathrm{~m}$, mientras que la altura máxima registrada es de $1,366 \mathrm{~m}$ y la altura mínima registrada es de -1,227 m (Secretaría de Comunicaciones y Transportes, 2008). Los datos del régimen anual de oleaje en aguas profundas muestran olas de más de $2,4 \mathrm{~m}$ con una frecuencia de ca. $21 \%-22 \%$ anual (Secretaría de Comunicaciones y Transportes, 2008). Los temporales que afectan esta costa con más frecuencia son los ciclones tropicales, en un promedio de seis anuales, pero solo pocos alcanzan la categoría de huracanes, y de estos últimos los de categoría uno son los más frecuentes (Hernández Unzón, 2008a; Hernández Unzón, 2008b). No existen datos, hasta donde se sabe, de los valores de sobreelevación marina por el efecto de los temporales, con excepción del evento El Niño en 1997, que registró sobreelevación marina de hasta $30 \mathrm{~cm}$ (Filinov et al., 2003). Durante el huracán Paulina de 1997 (considerado el huracán más importante del siglo en la costa norte del Pacífico este) no se registró rotura de la barras en este sector de la costa de Guerrero.

\section{¿Por qué estudiar la paleosismicidad de} la zona de subducción mexicana?

Basándose en las analogías y diferencias con otras zonas de subducción, se plantea la hipótesis de que asumiendo un periodo de tiempo de centenas a miles de años la zona de subducción mexicana es capaz de producir terremotos $(M \geq 9)$, con rupturas de falla de más de $800 \mathrm{~km}$ de largo (McCaffrey, 2007). La costa pacífica de México puede experimentar ambos: levantamiento y subsidencia durante grandes sismos. Por lo tanto, los grandes sismos que han producido deformación cosísmica deben haber dejado su huella en el registro geológico en la costa del Pacífico mexicano, como se observó con los sismos de $M>8$ que deformaron la costa en Jalisco en 1932 y en Michoacán en 1985 (Bodin \& Klinger, 1986; Corona-Esquivel et al., 1988; Cumming, 1933).

\section{Área de estudio}

Los estudios realizados en la laguna Mitla, en la costa de Guerrero, sugieren cambios relativos de nivel del mar en el Holoceno tardío indicativos de un probable terremoto e inundación marina ca. 3.400 A. P. (RamírezHerrera et al., 2007). Estudios posteriores realizados en la misma zona de marismas corroboran estos cambios ocurridos en Holoceno tardío y discuten la probable respuesta cosísmica e intersísmica de la costa en la zona de subducción mexicana, así como las discrepancias que existen entre estas (Ramírez-Herrera et al., 2009).

Esta investigación se realizó en la costa pacífica de México, en específico en el sector de la laguna de Mitla $\left(17^{\circ} 3^{\prime} \mathrm{N}\right.$ y $100^{\circ}$ $25^{\prime} \mathrm{W}$ ) (Figura $\mathrm{N}^{\circ} 2$ ). El objetivo de esta investigación es reexaminar las evidencias ambientales de cambios de nivel de la costa en base a los resultados obtenidos por Ramírez-Herrera et al. (2007 y 2009), utilizando un método multi-proxy (estratigrafía, microfósiles, polen, geoquímica, dataciones radiométricas) para discriminar entre las características de los depósitos de tsunami y los de alta energía del oleaje (tormentas), argumentar sobre probable ocurrencia de terremotos, y la respuesta de la costa a la deformación cosísmica.

\section{Métodos}

\section{Geomorfología}

Los humedales costeros pueden preservar el registro de cambios de nivel de la costa por su sensibilidad sedimentaria, y de flora y de fauna a los cambios de nivel del mar (cambios en las características de los sedimentos, polen, diatomeas, foraminíferos, ostrácodos; Shennan, 1999; Byrne et al., 2001; Horton et al., 2007). Por esta razón se utilizan imágenes de satélite (Landsat TM) y fotografías aéreas (escalas $1: 75.000$ y $1: 25.000$ ) para identificar sitios de humedales relativamente inalterados (Figura $\mathrm{N}^{\circ} 2$ 2). La selección de los sitios de muestreo con núcleos se hizo una vez concluida la cartografía de detalle de la geomorfología de los humedales y de las lagunas de Guerrero. 
Figura $\mathrm{N}^{\circ} 2$

Modelo del terreno muestra la localización de las lagunas Mitla, Coyuca y Tres Palos

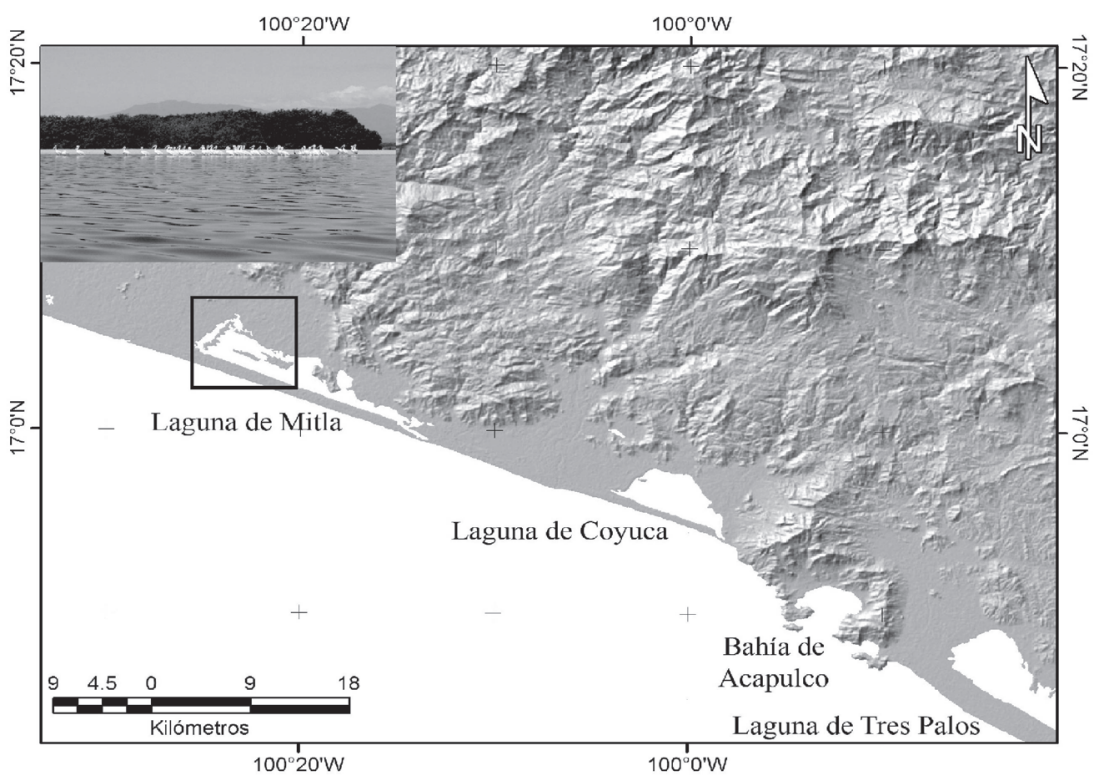

Recuadro indica localización de la Figura N³ 3 . La foto insertada muestra la laguna Mitla. Fuente: Elaboración propia.

\section{Estratigrafía}

Los cambios relativos de nivel del mar en la costa del Pacífico de México son el resultado tanto de los cambios absolutos del nivel del mar y los cambios locales en la elevación de la costa, resultantes de la repetición de sismos (subsidencia y levantamiento cosísmicos casi instantáneos) y de la deformación tectónica intersísmica de largo plazo. Por lo tanto, identificar los cambios relativos de nivel del mar registrados en los sedimentos costeros o comparar sitios con diferentes regímenes tectónicos, pueden proporcionar información valiosa sobre eventos sísmicos y tsunamis prehistóricos. La metodología para reconstruir estos eventos ha sido ya establecida y aplicada en diversos sitios en el mundo (Long \& Shennan, 1994; Islam \& Tooley, 1996; Zong \& Tooley, 1996; Cundy et al., 2000, entre otros). Dicha metodología incluye la recolec- ción de muestras por núcleos y/o trincheras, para identificar y fechar las unidades interestratificadas de sedimentos marinos y terrestres en la costa. En este estudio se colectaron una serie de núcleos (entre $\sim 2 \mathrm{~m}$ y $6 \mathrm{~m}$ de largo) usando una perforadora de vibración (vibracorer) Atlas Cobra. Aquí se discuten los resultados del registro realizado en la laguna Mitla (Figura № 2). Las coordenadas de cada lugar sondeado y sus elevaciones sobre el nivel del mar medio, se determinaron con la técnica de GPS diferencial usando receptores de doble frecuencia Leica SR520 y antenas AT504. Las estaciones de la red permanente de GPS SSN-Sismología-UNAM fueron usadas como referencia. Todas las estaciones de referencia tienen estimaciones de elevaciones actualizadas, basadas en el datum vertical de los mareógrafos de Acapulco y las estaciones GPS ACYA (Estación Acapulco Club de Yates GPS; Figura $N^{\circ} 1$ ). 
Cronología de sedimentos, geoquímica y análisis de microfósiles

La cronología de los sedimentos se determinó usando ${ }^{210} \mathrm{~Pb},{ }^{137} \mathrm{Cs}$ y ${ }^{14} \mathrm{C}$. Los sedimentos superficiales se fecharon usando ${ }^{210} \mathrm{~Pb}$ y ${ }^{137} \mathrm{Cs}$. Las submuestras se contaron durante ocho horas o más con un espectrómetro (Canberra well-type ultra-low background HPGe gamma ray), para determinar la actividad de ${ }^{210} \mathrm{~Pb}$ y ${ }^{137} \mathrm{Cs}$ y otros emisores de rayos gamma. Los límites de detección fueron ca. $3 \mathrm{~Bq} /$ kg. La datación con radiocarbono de muestras de madera y carbón se realizó en el Laboratorio del Acelerador de Espectrometría de Masa de la Universidad de Arizona y en el de Woods Hole, MA. Los valores de la fracción isotópica (delta ${ }^{13} \mathrm{C}$ ) se midieron en el laboratorio. Las calibraciones de radiocarbono se realizaron usando el programa CALIB 5.0 (Stuiver \& Reimer, 1993) y la base de datos IntCal04 (Reimer et al., 2004). La geoquímica de los sedimentos se determinó vía espectrofotómetro de masas acoplado a plasma inductivamente (ICP-MS, Perkin-elmer 6100DRC Unit), para determinar la distribución elemental en los sedimentos de los núcleos. Las muestras también fueron examinadas para detectar foraminíferos, ostrácodos y diatomeas. Las muestras para el análisis de foraminíferos y ostrácodos se lavaron en un tamiz de 63 $\mathrm{mm}$. Las muestras ricas en material orgánico se determinaron en húmedo, puesto que estos sedimentos tienden a formar una corteza orgánica sólida cuando están secos; todas las otras muestras, sin o con poco material orgánico, se contaron secas. El análisis del tamaño de las partículas y la caracterización de estas se hizo con un instrumento de difracción de rayos láser, Laser Beckman Couldter LS 230. La composición de los sedimentos se determinó con el estudio petrográfico, siguiendo a Kasper-Zubillaga et al. (1999), para derivar información del origen de los sedimentos y de su ambiente de depositación (Kasper-Zubillaga et al., 1999). La mayor parte de los sedimentos se examinaron para determinar la presencia de diatomeas utilizando un microscopio electrónico Leo S420.

\section{Resultados}

\section{Geomorfología}

El paisaje costero de la brecha sísmica de Guerrero se encuentra formado por una serie de barras de sistemas de lagunas. Las lagunas muestran extensas barras, áreas de marismas, planicies lodosas (mudflats), salinas, cordones litorales, playas, flechas y formas de tipo fluvial como deltas menores, valles, extensas planicies aluviales y terrazas fluviales (Figuras $\mathrm{N}^{\circ} 3, \mathrm{~N}^{\circ} 4$ y $\mathrm{N}^{\circ}$ 5).

La laguna costera Mitla está separada del mar por una barra formada a su vez por una serie de cordones litorales, aproximadamente dieciséis de estos, con altitud de hasta 6,5 $\mathrm{m}$ el más elevado. Algunos remanentes de estos cordones litorales forman una isla, isla Magueyes, dentro de la laguna. Mitla es una laguna somera de $1,6 \mathrm{~m}$ a $3 \mathrm{~m}$ de profundidad (Guzmán-Arroyo et al., 1986). Tiene $21 \mathrm{~km}$ de largo y $2 \mathrm{~km}$ aproximadamente de ancho. Esta laguna es receptora de aguas de escurrimiento y de ríos temporales (Páez-Osuna y Osuna-López, 1987), por lo que la salinidad de la laguna es muy baja, de ca. 3,5 ppt (Contreras-Espinosa \& Warner, 2004). El flujo de sedimentos aluviales a la laguna es limitado. La laguna se encuentra rodeada de manglares, marismas con pastos y salinas (Figuras $\mathrm{N}^{\circ} 3$ y $\mathrm{N}^{\circ} 4$ ). En total, se tomaron cuatro núcleos, tres de ellos en un transecto perpendicular a la costa, y otro más en posición perpendicular a dicho transecto (Figura $\mathrm{N}^{\circ} 3$ ). El primer núcleo se colectó entre la laguna y su barra (ACA-04-01), el segundo en la isla Magueyes (ACA-04-02), el tercero (ACA-04-06) en un ambiente de salinas (Figura $N^{\circ}$ 5). Por último, el cuarto núcleo se colectó en el límite entre la zona de manglar y la laguna (ACA-03-02).

\section{Estratigrafía}

Los cuatro núcleos colectados en la laguna Mitla muestran cambios evidentes con la profundidad (Figura $\mathrm{N}^{\circ} 6$ ). El núcleo uno (ACA-04-01), de 1,94 m de largo, fue tomado de un área de manglares en un lado de la barra y a 1,79 m sobre el nivel del mar. Este enseña una secuencia dominada por arena de color gris a gris-marrón, de tamaño fino a medio (2-5 f), con limo grueso (4-5 f), de $16 \mathrm{~cm}$ a $23 \mathrm{~cm}$ de profundidad (Figura $\mathrm{N}^{\circ} 6$ ). 
Figura $N^{\circ} 3$

Geomorfología y ambientes de deposición de la laguna Mitla

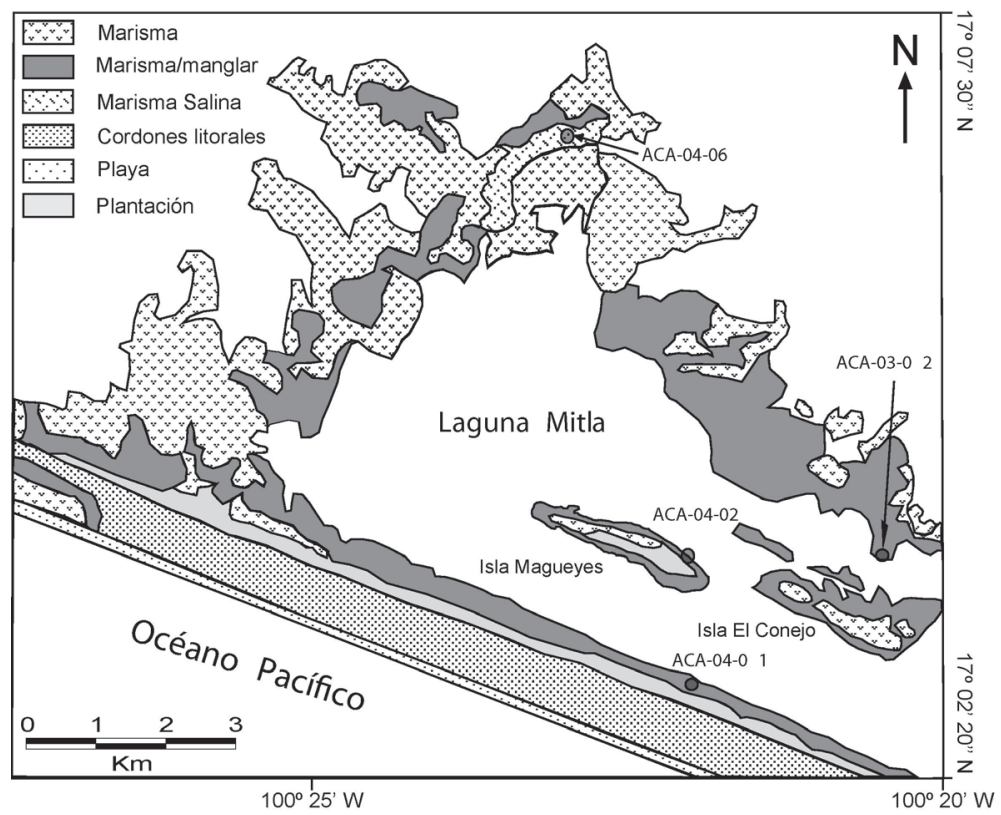

La localización de los sitios de muestreo de cada núcleo se muestra en letras negritas. Fuente: Modificada de Ramírez-Herrera et al., 2007.

Figura $\mathrm{N}^{\circ} 4$

Marisma en la laguna Mitla, Guerrero, sitio del núcleo ACA-04-06

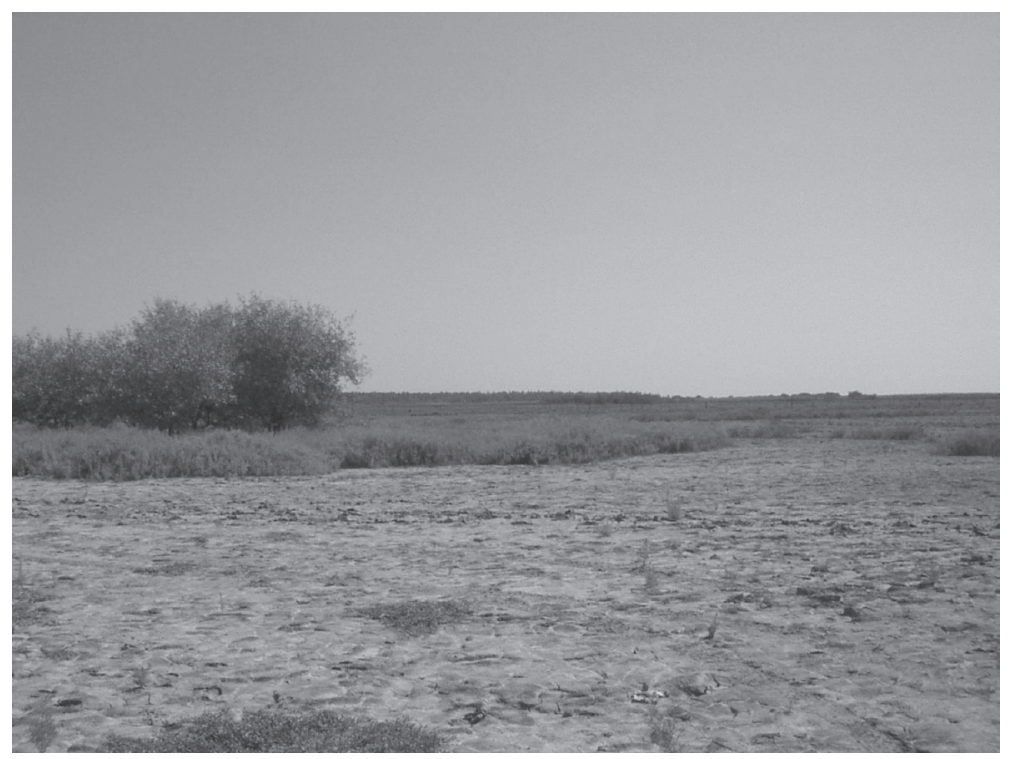

Fuente: Colección personal de la autora. 


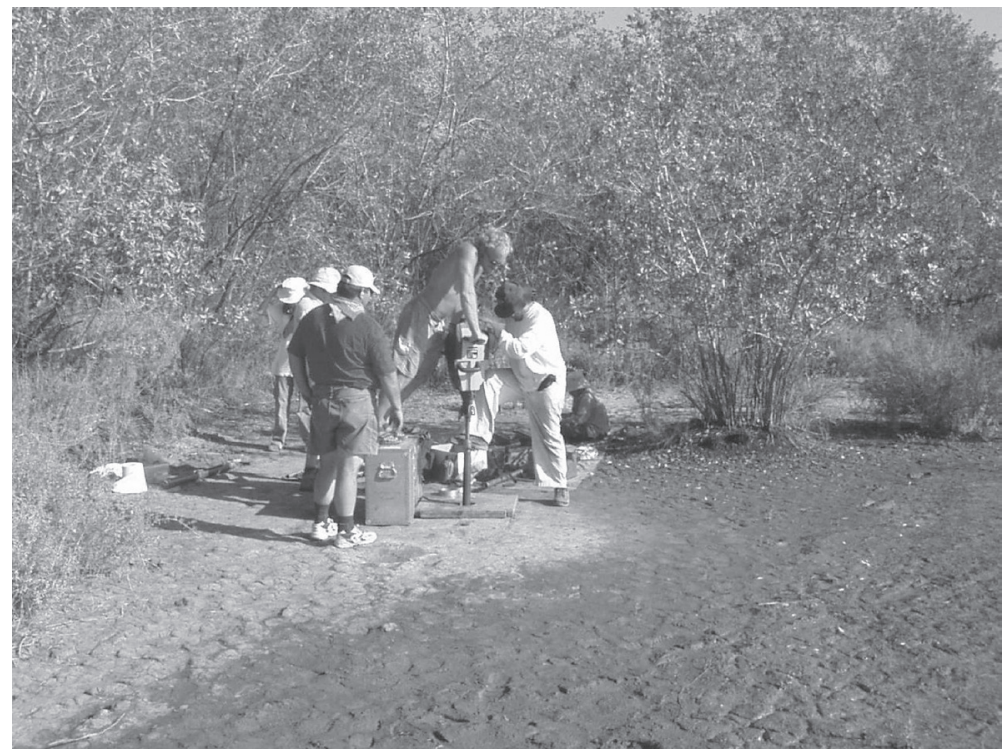

Fuente: Colección personal de la autora.

El núcleo dos (ACA-04-02), de 3,8 m de largo, fue tomado al lado norte de la isla Magueyes, a $0,81 \mathrm{~m}$ sobre el nivel del mar, siendo esta parte de un sistema de islas que se extiende discontinuamente a lo largo del eje noroeste-sureste de la laguna. También muestra una estratigrafía dominada por depósitos de arena de color marrón a gris-marrón, de tamaño medio ( 1 a $2 \mathrm{f}$ ), pasando los 3,5 m, con una unidad de arena media a muy fina, de los $129 \mathrm{~cm}$ a los $180 \mathrm{~cm}$ de profundidad.

El núcleo tres, de 3,99 $\mathrm{m}$ de longitud, (ACA-04-06), tomado en un área de salinas denominado Las Salinas, a 1,66 $\mathrm{m}$ sobre el nivel del mar, muestra limo y limo arcilloso marrón grisáceo a gris a los ca. 1,95 m. Bajo estos últimos hay arena interestratificada con limo arcilloso, suave, de color azul a grisazulado (posiblemente marino) entre los 1,95 $\mathrm{m}$ y los 3,2 $\mathrm{m}$ de profundidad. Desde los 240 $\mathrm{cm}$ hasta los $320 \mathrm{~cm}$ de profundidad, predomina arena gris interestratificada con limo arcilloso, mostrando un contacto basal abrupto, probablemente erosivo. Una unidad predominantemente de arena, con clastos rasgados (rip-up clasts) incorporados, que enseñan gradación normal, ocurre entre los $264 \mathrm{~cm}$ y $282 \mathrm{~cm}$ de profundidad. De los $320 \mathrm{~cm}$ a los $399 \mathrm{~cm}$ de profundidad, se presentan arcilla y limo laminado de color gris oscuro a negro grisáceo, con un alto contenido de materia orgánica ( $>80 \%$ pérdida por ignición), en forma de fragmentos de madera y materiales fíbricos bien preservados, posiblemente de vegetación de manglar.

El núcleo cuatro (ACA-03-02), de ca. 5,69 m de largo, fue tomado en un área de marismas en el límite norte de la laguna a 1,38 m sobre el nivel del mar. Este núcleo se caracteriza por su alto contenido en materia orgánica en la arcilla, principalmente en su porción basal, y la presencia de arena en la parte media-superior. La superficie del núcleo fue compactada y se perdió parte de los sedimentos (Figura $N^{\circ}$ 6). Hasta los $30 \mathrm{~cm}$ de profundidad predomina la arcilla limosa gris con abundante carbón, fragmentos de plantas y de madera (probablemente un depósito superficial de manglar). Entre los 40 $\mathrm{cm}$ y $45 \mathrm{~cm}$ de profundidad se observa arena 
media a gruesa, con contacto basal irregular separando arena media gris con motas de oxidación. Hasta los $104 \mathrm{~cm}$ de profundidad predominan arcilla limosa amarillo-rojiza (rica en óxidos de hierro), seca, y limo gris con concreciones de hierro. Entre los $132 \mathrm{~cm}$ y $136 \mathrm{~cm}$ de profundidad se observa arena gris, media a fina, con restos de plantas. De los $136 \mathrm{~cm}$ y hasta los $142 \mathrm{~cm}$ de profundidad la arena es gruesa con arcilla gris, poco clasificada, gradación normal, y se observa arena fina gris, limo y arcilla en la parte superior. De los $167 \mathrm{~cm}$ a los $220 \mathrm{~cm}$ de profundidad predomina la arcilla negra a gris muy oscuro con motas amarillas alternada con limo. A partir de los $229 \mathrm{~cm}$ de profundidad y hasta la base del núcleo, $569 \mathrm{~cm}$ de profundidad, predomina la alternancia de arcilla negra, con variaciones a gris, muy rica en materia orgánica formada por fragmentos

Figura $N^{\circ} 6$

Estratigrafía y correlación de los núcleos ACA04-01, ACA-04-02 y ACA-04-06 y ACA-03-02, colectados en la laguna Mitla. La elevación de los núcleos con respecto al nivel del mar fue medida con GPS diferencial

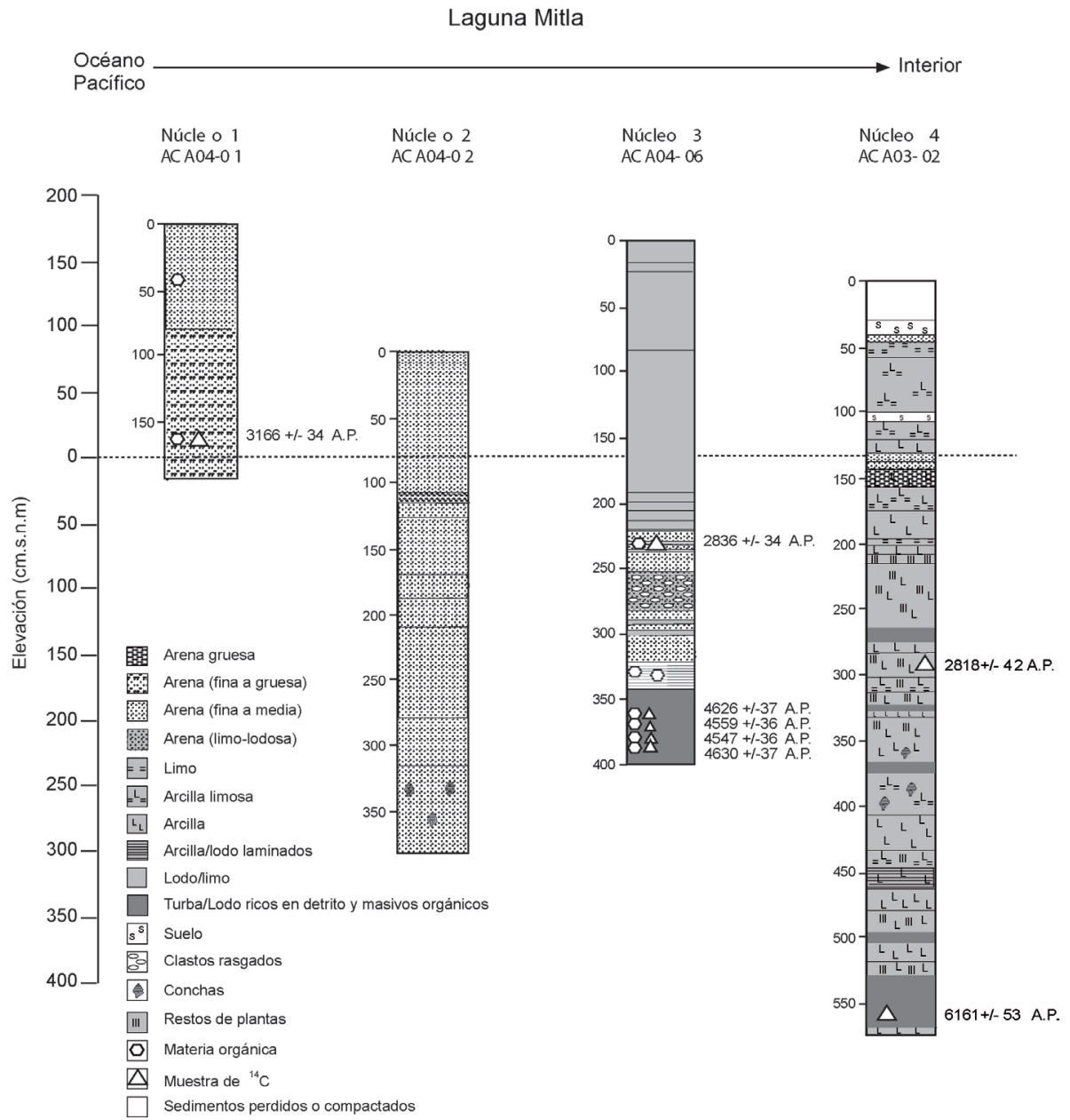

Fuente: Modificada de Ramírez-Herrera et al., 2007; Ramírez-Herrera et al., 2009. 
grandes de madera y materiales fíbricos bien preservados, posiblemente de vegetación de manglar. El contacto basal parece abrupto a los $280 \mathrm{~cm}$ de profundidad. Entre los $280 \mathrm{~cm}$ y $332 \mathrm{~cm}$ de profundidad se observan restos de carbón y turba negra, y entre los $332 \mathrm{~cm}$ y los $404 \mathrm{~cm}$ de profundidad conchas y restos de plantas. El contacto basal a los $447 \mathrm{~cm}$ de profundidad es aparentemente abrupto, probablemente erosivo. Entre los $447 \mathrm{~cm}$ y los $456 \mathrm{~cm}$ de profundidad la arcilla de color marrón a grisáceo está finamente laminada (Figura $\mathrm{N}^{\circ} 6$ ).

El análisis petrográfico de los núcleos uno, dos y tres muestra la predominancia de cuarzo, feldespatos y micas, con algunos metales pesados en las unidades de grano grueso. La arena en el núcleo uno está bien clasificada pero poco entre los $16 \mathrm{~cm}$ y 23 $\mathrm{cm}$. De los $23 \mathrm{~cm}$ hacia la base, la arena está moderadamente a poco clasificada. Esto se puede explicar por la cercanía del núcleo a la barra, donde tienen lugar diferentes procesos (eólicos y de laguna) que producen una interdigitación en un ambiente heterogéneo. El núcleo dos muestra una clasificación pobre de la arena hacia la superficie, siendo que esta se vuelve moderadamente a muy pobremente clasificada de los $129 \mathrm{~cm}$ a los $180 \mathrm{~cm}$ de profundidad. La arena y el limo interestratificados muestran una clasificación predominantemente de pobre a moderada. La variación en el tamaño de las partículas (arena media a muy fina, y limo) y la clasificación media a pobre, sugieren que estos sedimentos han sido sujetos a condiciones de energía alta. Por otro lado, el núcleo cuatro indica de manera predominante arcilla y limo con algo de arena intercalada. Lo primero señala ambientes de baja energía con eventos esporádicos de alta energía.

En general, el sedimento inorgánico es el componente primario de los núcleos, con pocas unidades ricas en materia orgánica y fragmentos de plantas dispersos en todos estos, con excepción del núcleo cuatro; este último formado predominantemente por sedimento orgánico. Todos los núcleos contienen fragmentos de concha. A su vez, el carbón está presente en todos los núcleos, indicando la quema local de madera o desecación y pedogénesis (Cundy et al., 2000). En particular, los núcleos tres y cuatro muestran contactos abruptos entre las unidades sedimentarias, lo que indica cambios rápidos de condiciones de depositación a periodos de erosión. Sin embargo, las unidades sedimentarias individuales no se pueden trazar entre los núcleos, mostrando una estratigrafía heterogénea a través de la laguna. Estas variaciones en la estratigrafía de cada núcleo podrían reflejar los diferentes ambientes de la laguna. Como se señala arriba, el núcleo uno representa el ambiente cercano a la barra, mientras que los núcleos tres y cuatro representan ambientes de baja energía con eventos esporádicos de alta energía. En particular, los núcleos tres y cuatro son los que muestran contactos abruptos entre unidades que evidencian cambios de gran escala en ambientes de depositación que pueden estar relacionados con cambios locales de nivel del mar. Estos dos núcleos fueron seleccionados para análisis geoquímicos y de microfósiles.

\section{Geoquímica de elementos traza y elementos mayores}

Los núcleos tres y cuatro muestran variaciones claras a profundidad en la geoquímica de los sedimentos. Los cambios en la composición de los elementos mayores y traza son claros y acordes con la estratigrafía identificada.

\section{Geoquímica núcleo tres}

En el núcleo tres, entre los $320 \mathrm{~cm}$ y 399 $\mathrm{cm}$ de profundidad (unidad de arcilla limosa rica en material orgánico), no se observa enriquecimiento de elementos traza a pesar de su naturaleza altamente orgánica. Esto podría indicar condiciones de baja salinidad (y por tanto la formación menor de fases de sulfuros de diagénesis temprana) en dicha unidad y que coincide con las especies epífitas dominantes de agua dulce de las diatomeas presentes (ver más adelante la sección de microfósiles). La geoquímica y las diatomeas, la presencia de material fíbrico bien preservado, y los fragmentos de madera en esta unidad, señalan un probable ambiente salobre en la margen de la laguna.

En la unidad entre los $190 \mathrm{~cm}$ y $240 \mathrm{~cm}$ de profundidad (limo arcilloso, arcilla limosa azul oscura, arena interestratificada y limo arcilloso con materia orgánica) se presenta un 
aumento significativo de elementos traza $(\mathrm{Ca}$, As, Ni, Sb, Sn y de Cs) (Figura No 7a). Sin embargo, no hay enriquecimiento en esta unidad de los elementos $\mathrm{Al}$, K y Rb, usados comúnmente como indicadores de entrada de sedimentos de siliclastos finos (Zwolsman et al., 1993; Cundy et al., 2006). Esto muestra que el aumento de elementos traza $(\mathrm{Ca}, \mathrm{As}, \mathrm{Ni}, \mathrm{Sb}$ y $\mathrm{Sn}$ ) no es un efecto del cambio en el tipo de sedimento o de su procedencia. Por el contrario, el enriquecimiento puede ser debido a la formación de fases secundarias (diagénesis temprana), de fases minerales, particularmente de sulfuros (en el caso de As, Ni, Sb y Sn) y de carbonatos (en el caso del Ca), con los que estos elementos pueden estar fuertemente asociados. La formación de sulfuros secundarios (y de carbonatos) es un proceso común en los sedimentos relativamente ricos en orgánicos salobres a marinos (Zwolsman et al., 1993; Spencer et al., 2003; Cundy et al., 2006), indicando en este horizonte un ambiente de depositación salobre a marina. No es clara la causa del enriquecimiento en Cs en este horizonte. Los niveles altos en Cs son generalmente indicativos de sobreconcentración por removilización de limo y coinciden precisamente con los niveles de alta energía.

Figura $\mathrm{N}^{0} 7$

Concentraciones de elementos de los análisis de sedimentos usando ICP-MS en los núcleos (a) ACA-04-06 y (b) ACA-03-02

a)
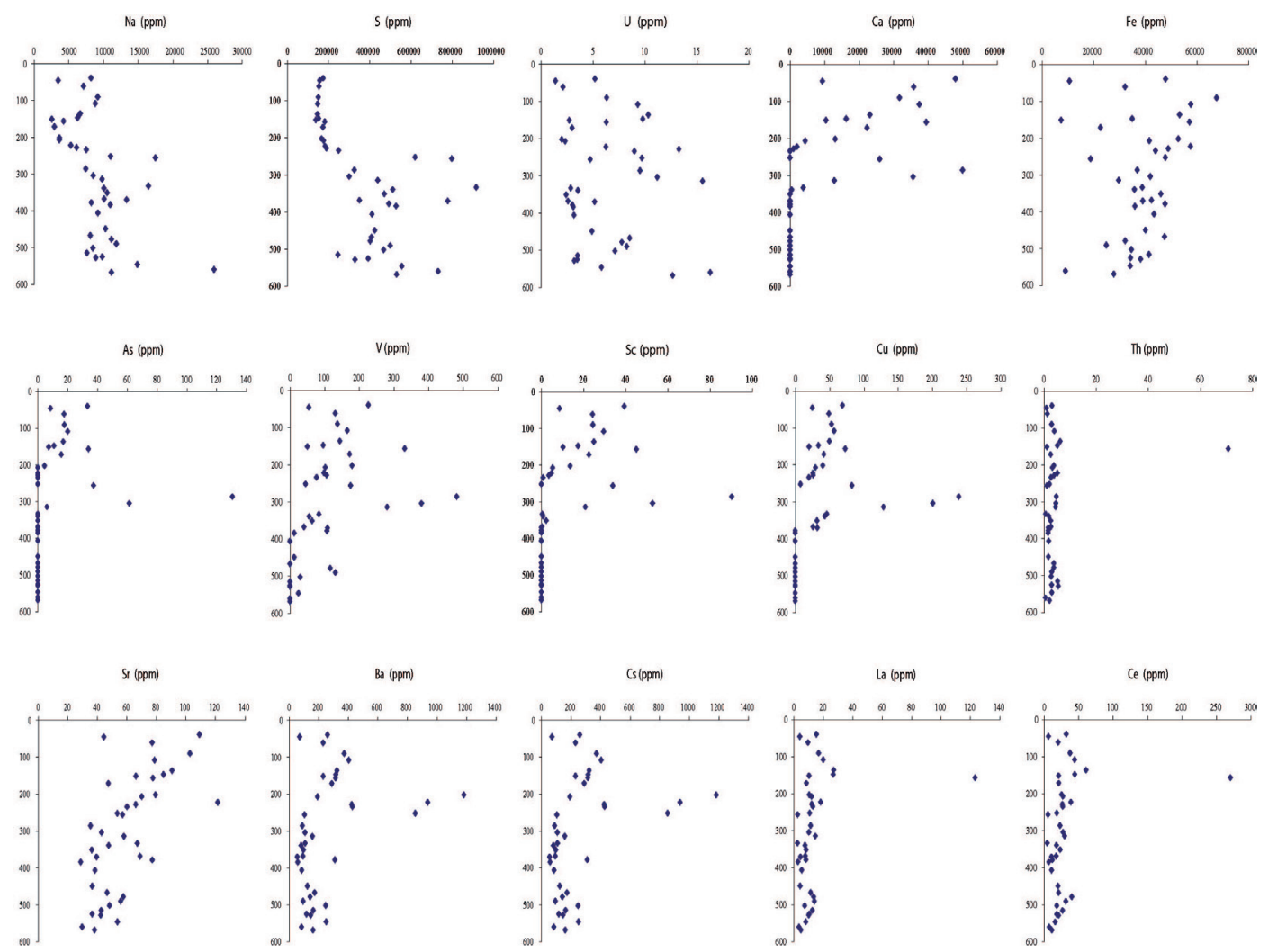
b)
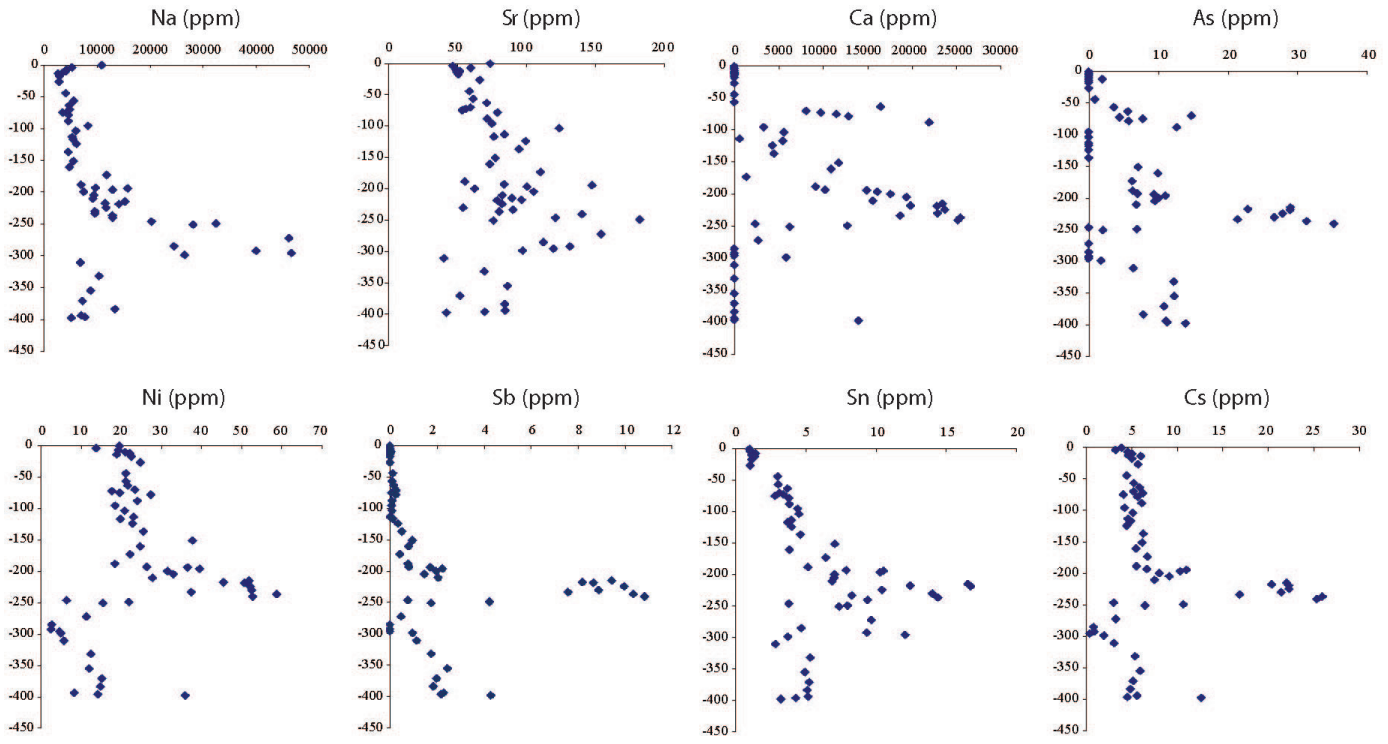

Fe (ppm)

Ti (ppm)
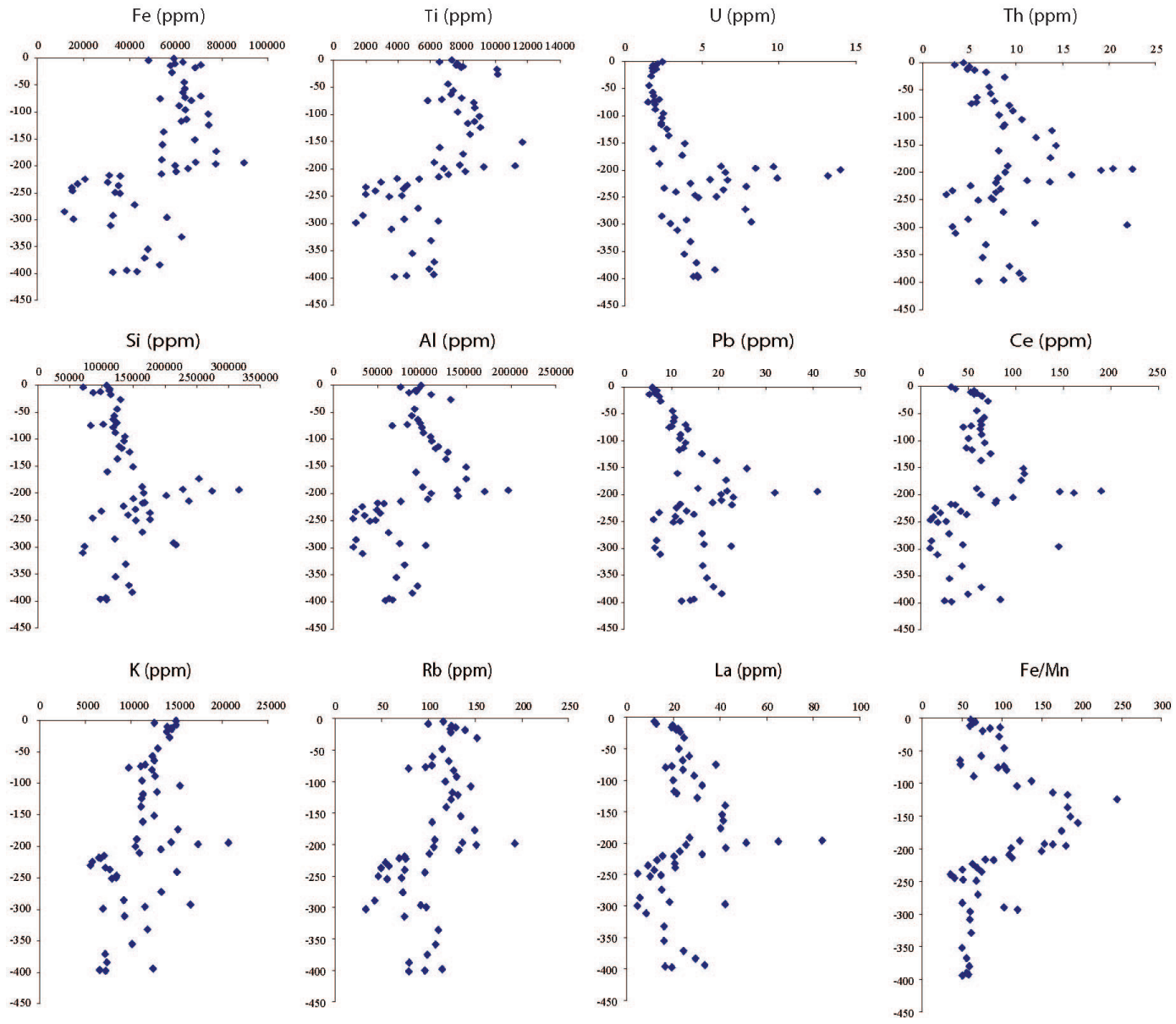

Fuente: Modificada de Ramirez-Herrera et al., 2007; Ramírez et al., 2009. 
La arena gris y la arcilla que se depositan por arriba de estas unidades, ca. $230 \mathrm{~cm}$ de profundidad, son geoquímicamente distintas, careciendo de los elementos antes mencionados y de $\mathrm{Cu}$ y $\mathrm{Zn}$ (estos dos no se muestran en la Figura $\mathrm{N}^{\circ} 7 \mathrm{a}$ ), pero enriquecidos en $\mathrm{Na}$ $y$, en menor grado, en Sr (Figura $\mathrm{N}^{\circ} \mathrm{7a}$ ). El aumento en las concentraciones de $\mathrm{Na}$ ha sido planteado en varios estudios como indicador de inundación marina (Chagué-Goff et al., 2002).

A los $195 \mathrm{~cm}$ de profundidad (tope de la unidad de arcilla azul) se presentan concentraciones máximas de $\mathrm{Fe}, \mathrm{Ti}, \mathrm{U}, \mathrm{Th}, \mathrm{Si}, \mathrm{Al}, \mathrm{Pb}$, $\mathrm{Ce}, \mathrm{K}, \mathrm{Rb}$ y La (Figura $\mathrm{N}^{\circ} 7 \mathrm{a}$ ), señalando un probable cambio en la procedencia de los sedimentos (incluyendo un aumento de entrada de minerales pesados) a esta profundidad. Es también notable un enriquecimiento similar en $\mathrm{Ca}, \mathrm{Sb}, \mathrm{As}, \mathrm{Ni}$ y en otros elementos calcógenos, que en el observado en las unidades entre los $190 \mathrm{~cm}$ y los $240 \mathrm{~cm}$ de profundidad.

En la parte superior del núcleo $(0 \mathrm{~cm}$ a $190 \mathrm{~cm}$ de profundidad) la geoquímica de sedimentos es uniforme (con la excepción del Ca y el As). El radio de Fe a Mn, que ha sido utilizado como un indicador de cambios en las condiciones redox de captación de las lagunas y los lagos (Vorren \& Alm, 1999; Virkanen, 2000), muestra un importante aumento entre los $100 \mathrm{~cm}$ a $200 \mathrm{~cm}$ de profundidad (Figura $\mathrm{N}^{\circ} 7 \mathrm{a}$ ), reflejando una disminución potencial del Eh en las áreas terrestres adyacentes, causada por la inundación de los suelos.

\section{Geoquímica núcleo cuatro}

El Na y $\mathrm{S}$ (el último en forma de sulfatos y en forma de sulfuros secundarios, diagenéticos, generados bajo condiciones salobres a marinas) han sido propuestos como indicador de paleo-salinidad y de incursión marina en varios estudios de sedimentos costeros (López-Buendía et al., 1999; Cundy et al., 2006). En el núcleo cuatro, a los $561 \mathrm{~cm}$, $370-380 \mathrm{~cm}, 330 \mathrm{~cm}$ y $260 \mathrm{~cm}$ de profundidad, las concentraciones de $\mathrm{Na}$ y $\mathrm{S}$ muestran varios máximos subsuperficiales, que podrían reflejar un aumento en la paleo-salinidad y posibles incursiones marinas. Dado el color oscuro de los sedimentos, una proporción significativa del $\mathrm{S}$ medido es probable que se encuentre en la forma de sulfuros secundarios (diagenéticos), generados por la reducción microbiana de sulfatos del agua marina en el tiempo.

A los $340 \mathrm{~cm}$ contiene poca concentración de Ca, y en particular entre los $223 \mathrm{~cm}$ y $253 \mathrm{~cm}$ de profundidad, que es muy variable entre los 5 wt \% y cero, a lo largo del núcleo (Figura $\mathrm{N}^{0} 7 \mathrm{~b}$ ). Los cambios de concentración en la mayoría de los elementos analizados coinciden con aquellos de la estratigrafía. A título ilustrativo, entre los $148 \mathrm{~cm}$ y $223 \mathrm{~cm}$ de profundidad, la marcada reducción en elementos como $\mathrm{Na}, \mathrm{S}, \mathrm{U}, \mathrm{As}, \mathrm{V}, \mathrm{Sc}$ (Figura $N^{\circ} 7 b$, probablemente refleja el aumento en cuarzo de la arena en este intervalo. A los $157 \mathrm{~cm}$ de profundidad los elementos Th, $\mathrm{La}$ y $\mathrm{Ce}$ (y en menor grado $\mathrm{Ti}, \mathrm{Cr}, \mathrm{Ni}, \mathrm{Sc}, \mathrm{V}$ y $\mathrm{Fe}$ ) muestran una concentración máxima prominente que puede reflejar un pulso en el aumento de minerales pesados durante la depositación de arena.

Además, a los $287 \mathrm{~cm}$ de profundidad se observan concentraciones máximas de As, $\mathrm{V}, \mathrm{Cu}, \mathrm{Ca}$ y $\mathrm{Sc}$ (y menos en $\mathrm{Ni}$ y $\mathrm{Cr}$ ). El enriquecimiento en $\mathrm{Sc}$, que es inherente a las reacciones redox, imposibilita la explicación de procedencia de diagénesis temprana en el aumento observado en la concentración de estos elementos. En cambio, estas concentraciones máximas, a esta profundidad, pueden ser el resultado de procesos pedogenéticos y de laterización, con Fe y Al, mostrando también un ligero enriquecimiento.

A los $142 \mathrm{~cm}$ y $151 \mathrm{~cm}$, y a los $40 \mathrm{~cm}$ de profundidad, que coincide con los horizontes de arena, el $U$ y Ca muestran concentraciones ligeramente enriquecidas. El análisis de los datos de geoquímica utilizando el Análisis de Componentes Principales, subraya la fuerte asociación entre As, V, Cu y Sc, y también discrimina entre $\mathrm{Na}$ y $\mathrm{S}$ de otros elementos (Figura $\mathrm{N}^{\circ} 7 \mathrm{~b}$ ).

\section{Microfósiles}

Las muestras de cada unidad estratigráfica identificadas en el núcleo tres fueron examinadas para detectar foraminíferos y trazas de ostrácodos/conchas. No se encontró evidencia alguna. Su ausencia podría ser el 
resultado de disolución posdepositacional. Una alta entrada de ácidos orgánicos se observó anteriormente en la laguna Mitla durante la temporada de lluvias (González Villaseñor, 1977), que puede inhibir el crecimiento como la conservación de pruebas de ostrácodos y conchas calcáreas. Debido a la ausencia de microfósiles calcáreos, se usaron muestras seleccionadas de sedimentos del núcleo tres para identificar microfósiles silíceos (diatomeas). Las diatomeas fueron intermitentemente preservadas en el núcleo tres, por tal razón no se representan gráficamente. Las muestras $3 \mathrm{~cm}$ a $7 \mathrm{~cm}, 7 \mathrm{~cm}$ a $9 \mathrm{~cm}$, y $396 \mathrm{~cm}$ a $397 \mathrm{~cm}$ de profundidad, contienen diatomeas, y la muestra de $21 \mathrm{~cm}$ a $34 \mathrm{~cm}$ de profundidad, contiene algunas espículas de esponja sin diatomeas. Hacia la base del núcleo, la muestra a 396 cm a 397 cm de profundidad, contenía abundantes formas epífitas de agua fresca, representando un margen de laguna de agua dulce o un estanquillo superficial con abundantes plantas acuáticas. Las especies presentes incluyen Cocconeis placentula var. lineate, Synedra goulardii, Navicula mutica y variedades de
Epithemia y Gomphonema. En contraste, las muestras de $3 \mathrm{~cm}$ a $7 \mathrm{~cm}$ y de $7 \mathrm{~cm}$ a $9 \mathrm{~cm}$ de profundidad, tuvieron pocas diatomeas, y fueron estuarinas, bénticas (ejemplo, Cocconeis placentula var. lineate, Synedra goulardii, Navicula mutica y variedades de Epithemia y Gomphonema). La arena y limo arcilloso azul no contenían diatomeas (Ramírez-Herrera et al., 2007). El núcleo cuatro fue examinado para detectar la presencia de microfósiles. Los resultados de estos análisis revelan la presencia de diatomeas, polen y fitolitos. Estos resultados han sido reportados en otro trabajo (Kennett et al., 2004), de los se hará referencia en la sección de interpretación.

\section{Datación radiométrica}

La datación con ${ }^{210} \mathrm{~Pb}$ y ${ }^{137} \mathrm{Cs}$ se intentó en los $70 \mathrm{~cm}$ superiores del núcleo tres, señalando que la parte superior de la secuencia pudo haber sido removida por alteración antropogénica. No se detectó ${ }^{137} \mathrm{Cs}$ en ninguna de las muestras, mientras que el ${ }^{210} \mathrm{~Pb}$ es o está cerca del límite de la actividad (se estimó usando ${ }^{214} \mathrm{~Pb}$ ) en los $70 \mathrm{~cm}$ analizados. Cin-

\section{Cuadro $N^{\circ} 1$}

Edades de radiocarbono de las muestras de los núcleos ACA-04-06 y ACA-03-02 de los sitios de estudio en laguna Mitla

\begin{tabular}{|l|c|c|c|c|c|c|}
\hline $\begin{array}{c}\text { Número de } \\
\text { muestra del } \\
\text { laboratorio }\end{array}$ & Núcleo & $\begin{array}{c}\text { Profun- } \\
\text { didad } \\
(\mathrm{cm})\end{array}$ & $\begin{array}{c}\text { Material } \\
\text { fechado }\end{array}$ & ${ }^{14}$ C a.P. & $\begin{array}{c}\text { Años calibrados } \\
\text { (rango 2 sigma) }\end{array}$ & $\begin{array}{c}\text { Edad calibrada } \\
\text { (rango 2 sigma) } \\
\text { Cal a.C./d.C. }\end{array}$ \\
\hline AA59462 & ACA-04-01 & 155 & Madera & $3.166+/-34$ & $3.336-3.456$ & $1.507-1.387$ \\
AA59469 & ACA-04-06 & 394 & $\begin{array}{c}\text { Carbón / } \\
\text { madera }\end{array}$ & $4.630+/-37$ & $5.297-5.467$ & $3.518-3.393$ \\
AA59470 & ACA-04-06 & 387 & Madera & $4.547+/-36$ & $5.051-5.317$ & $3.243-3.102$ \\
AA59471 & ACA-04-06 & 377 & Madera & $4.559+/-36$ & $5.053-5.437$ & $3.240-3.104$ \\
AA59472 & ACA-04-06 & 371 & Madera & $4.626+/-37$ & $5.294-5.468$ & $3.519-3.345$ \\
AA59474 & ACA-04-06 & 234 & Madera & $2.836+/-34$ & $2.859-3.063$ & $1.092-910$ \\
AA54043 & ACA-03-02 & $299-300$ & Carbón & $2.818+/-42$ & $2.842-3.043$ Cal BP & $1.094-893$ \\
AA54044 & ACA-03-02 & $560-564$ & Madera & $6.161+/-53$ & $6.901-7.176$ Cal BP & $5.227-4.952$ \\
\hline
\end{tabular}

(1) NSF-Arizona AMS Facility y NOSAMS AMS Laboratory (núcleo ACA-03-02).

Las calibraciones de radiocarbono se realizaron usando el programa CALIB 5.0 (Stuiver and Reimer, 1993) y la base de datos de IntCal04 (Reimer et al., 2004).

Fuente: Modificada de Ramírez-Herrera et al., 2007; Ramírez et al., 2009. 
co muestras del núcleo tres, dos del núcleo cuatro y una del núcleo uno fueron fechadas con ${ }^{14} \mathrm{C}$ (Cuadro $\mathrm{N}^{\circ}$ 1) (Ramírez-Herrera et al., 2007; Ramírez et al., 2009); una muestra de un carbón grande $(1 \mathrm{~cm}$ de largo) tomada de la unidad de arcilla gris oscura a negra, rica en materia orgánica, a los $394 \mathrm{~cm}$ de profundidad del núcleo tres, y tres muestras de madera a los $387 \mathrm{~cm}, 377 \mathrm{~cm}, 371 \mathrm{~cm}$ de profundidad en la arcilla gris oscuro de la base del núcleo, y una muestra de madera de la unidad de arcilla azul a los $234 \mathrm{~cm}$ de profundidad. Las cuatro muestras de la base del núcleo dieron edades que se sobreponen y podrían reflejar la mezcla o la depositación instantánea de este material.

Con el propósito de discutir y realizar correlaciones con fechas de otros estudios publicados de la región (aquí citados), se usarán las edades en años A. P. no calibradas (las fechas calibradas se presentan en la Cuadro $\mathrm{N}^{\circ}$ 1). La muestra a los $234 \mathrm{~cm}$ de profundidad dio una edad de $2.836 \pm 34$ A. P. Las edades estimadas del núcleo tres indican que esta secuencia tiene hasta $4.626 \pm 37$ A. P. (Ramírez-Herrera et al., 2007). Dos muestras del núcleo cuatro se fecharon con ${ }^{14} \mathrm{C}$, un fragmento grande de madera $(1,5 \mathrm{~cm}$ de largo) en la turba negra de la base del núcleo, y la otra, un fragmento de carbón a los 299-300 $\mathrm{cm}$ de profundidad, en la unidad de arcilla gris oscuro (Cuadro $N^{\circ} 1$ ). Debido a que los fragmentos fechados provienen de detritos, la edad de las muestras puede preceder a la depositación por un número desconocido de años, por lo que se seleccionaron fragmentos que tenían menos probabilidad de estar retrabajados. Estos fragmentos muy probablemente derivaron de vegetación in situ. La muestra de carbón a los 299-300 cm de profundidad, dio $2.818 \pm 42$ A. P. La muestra de la base del núcleo (560-564 cm de profundidad) dio una edad de $6.161 \pm 53$ A. P. (Ramírez-Herrera et al., 2007; Ramírez et al., 2009). Esto significa que las secuencias completas de los núcleos analizados de la laguna Mitla fueron depositadas desde que la tasa de ascenso posglacial del nivel del mar había disminuido globalmente durante el Holoceno medio. No existen estimaciones del nivel relativo del mar para el área inmediata. Sin embargo, datos de otros lugares de la costa del Pacífico en México y de California en Estados Unidos, indican que la tasa de ascenso regional del nivel del mar declinó entre los ca. 6.000 y $5.200 \mathrm{~A}$. P. (Curray et al., 1969; Atwater et al., 1977; Sirkin, 1985). En base a las edades de radiocarbono de las muestras de la laguna Mitla, la tasa neta de sedimentación desde los $6.161 \pm$ 53 A. P. es de ca. 1 mm/año (Ramírez-Herrera et al., 2009). No obstante, se debe señalar que dicha tasa promedio es probable que enmascare variaciones considerables en las tasas de acumulación/erosión, basándose en los cambios sedimentológicos que se observan en los núcleos tres y cuatro, y a los contactos abruptos entre las unidades (Figura $N^{\circ} 4$ ), que señalan episodios de erosión o cambios rápidos en el nivel de elevación de la costa (por ejemplo episodios tectónicos).

\section{Discusión y consideraciones finales}

\section{Cambios en la elevación de la costa e incursión marina prehistóricos}

El nivel del mar estaba cerca de su nivel actual para ca. 6.000 y 5.000 A. P. en la costa de California (Atwater et al., 1977), en el noroeste de la costa del Pacífico de México y en el Golfo de California (Curray et al., 1969; Sirkin, 1985; Ortlieb, 1986; Caballero et al., 2005). La estratigrafía de la laguna Mitla indica que la barra costera se estableció (lo que aisló a la laguna Mitla de la depositación de alta energía) y la laguna se formó ca. 6.000 A. P., así como otras lagunas costeras en la región (Curray et al., 1969; GonzálezQuintero, 1980; Sirkin, 1985; Caballero et al., 2005). La estratigrafía de la laguna muestra que por lo menos para ca. 4.630 A. P., la barra había concluido su formación en la laguna Mitla y que para los ca. 4.660 A. P., sucedió lo mismo en la adyacente laguna Coyuca (Ramírez et al., 2005).

Las capas de arena dispuestas sobre la turba salobre de manglar, en el núcleo tres, parecen estar asociadas a un evento de inundación marina, ya sea por un cambio relativo de nivel del mar, por tsunami o por una tormenta (Ramírez-Herrera et al., 2007). Las características de los depósitos de arena-gradación normal, más finos hacia arriba, laminaciones internas de los lodos, contacto basal abrupto y erosional, y particularmente la presencia de clastos rasgados incorporados a la arena (Fi- 
gura $\mathrm{N}^{\circ} 4$ ) son similares a las de depósitos de tsunami descritas en otros trabajos (Morton et al., 2007; Kortekaas \& Dawson, 2007; Nichol et al., 2007). La información geoquímica para los núcleos tres y cuatro indica cambios significativos en las condiciones ambientales del medio, en la procedencia de los sedimentos y/o en la actuación de procesos diagéneticos, que pueden reflejar la influencia de cambios relativos de nivel del mar (Ramírez-Herrera et al., 2007). El aumento en los indicadores de paleo-salinidad (concentración de elementos, micro y macrofósiles) señalan una incursión marina provocada por un evento de alta energía en torno a los ca. 3.400-3.500 A. P. (edad por extrapolación). Asumiendo que el nivel del mar era estable localmente en ese momento y que la barra ya estaba formada en ese mismo tiempo (Curray et al., 1969; Sirkin, 1985; Ortlieb, 1986; Caballero et al., 2005), entonces es muy probable que la causa de este cambio rápido en la salinidad, de condiciones de agua dulce/salobre a condiciones marinas, fue provocado por una incursión marina a la marisma salobre (Figura $N^{\circ}$ 8). Además, las evidencias geoquímicas y

Figura $\mathrm{N}^{\circ} 8$

Paleo-ambientes, interpretación de la deformación tectónica de la costa e incursiones marinas en la zona de la brecha sísmica noroeste de Guerrero durante el Holoceno tardío, a partir del núcleo cuatro

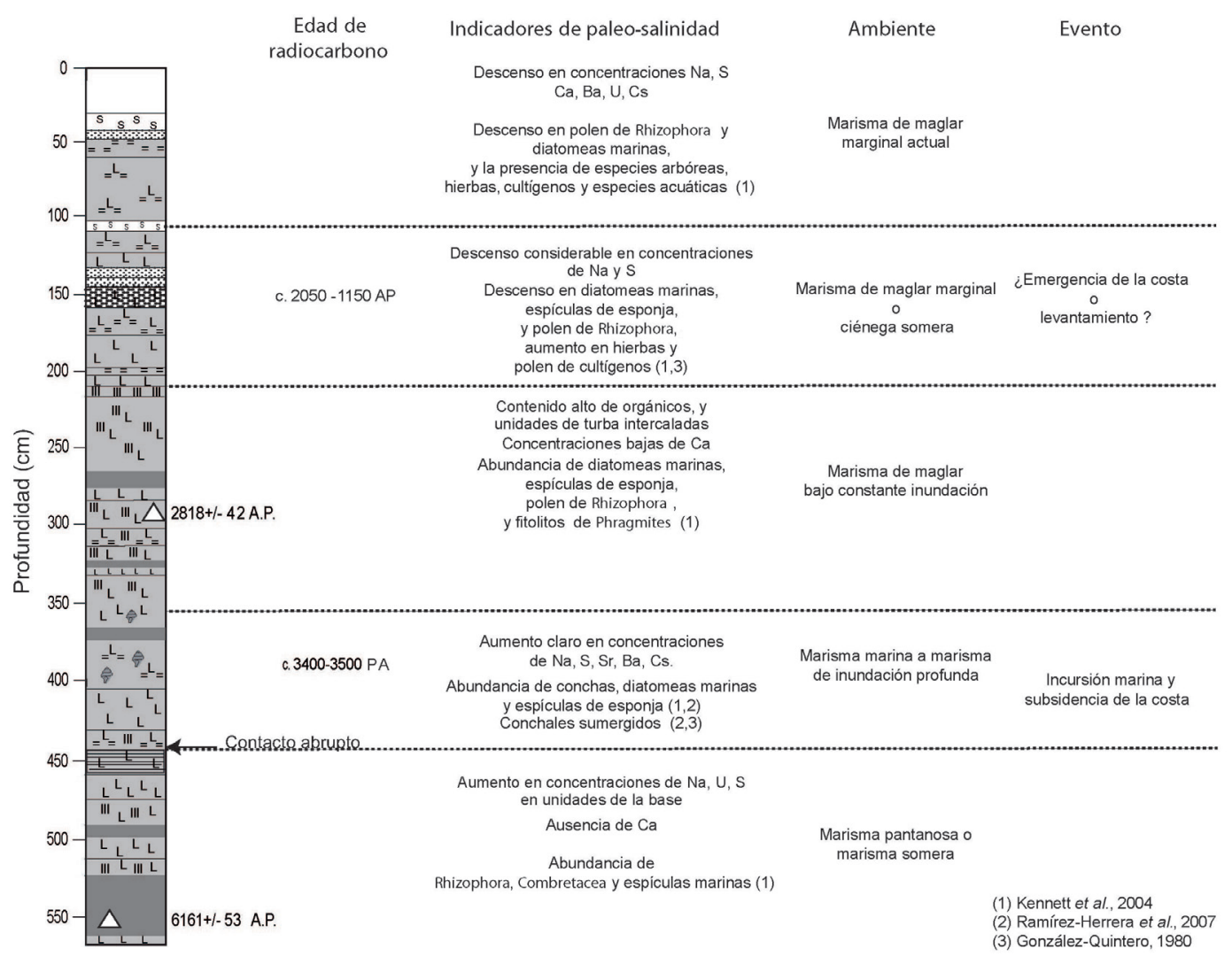

La barra esquemática en la margen izquierda, no está a escala, muestra los cambios de nivel de la costa como respuesta a la tectónica de la zona de subducción. El signo negativo indica subsidencia y el positivo levantamiento.

Fuente: Modificada de Ramírez-Herrera et al., 2009. 
la distancia del sitio de estudio a la barra $(\sim 5$ $\mathrm{km})$, sugieren una incursión marina, inducido por actividad sísmica y no por una tormenta. Sumado a lo expuesto, los datos históricos sugieren que los sismos son frecuentemente acompañados por cambios en el nivel de la costa (Bodin \& Klinger, 1986) y tsunamis a lo largo de la costa del Pacífico mexicano (Farreras, 1997). Por ejemplo, el evento de 1925 produjo una ola de 7-11 m cerca de Zihuatanejo, Guerrero; mientras que una ola de tsunami de 3-8 $\mathrm{m}$ de altura fue registrada en 1782 en Acapulco, Guerrero. Evidencias históricas de un gran sismo $(M \sim 8,5)$ ocurrido en la costa vecina de Oaxaca en 1787, ca. $200 \mathrm{~km}$ al sur de Acapulco, indican un gran tsunami que penetró ca. $6 \mathrm{~km}$ tierra adentro (Núñez-Cornú et al., 2008; Suárez, 2007). Sin embargo, estos datos no muestran información referente a alguna señal discernible, por ejemplo, sedimentos depositados entonces por estos tsunamis. La probabilidad de un tsunami y su probable extensión como causa de este evento aún tiene que probarse con más núcleos y trincheras en la zona de marismas de la laguna Mitla y otras en la costa de Guerrero. Se cree, sin embargo, que los cambios en salinidad y las capas de arena no parecen atribuirse a la rotura de la barra por tormentas excepcionales (Wang \& Horwitz, 2006), o por un tsunami, sin subsidencia (y sin la restauración de la barra por la corriente litoral), la laguna sería más salobre/marina en un plazo largo de tiempo, pero (a) la rotura en la barra debería de ser muy grande para provocar este cambio, y (b) se esperaría entonces un cambio a aguas de mar abierto, facies de costa abierta; algo que no se observa en los núcleos.

El retorno gradual a un ambiente menos salino, de marisma de manglar, se observa por el cambio gradual en la composición química de los sedimentos y de los microfósiles que sugieren un ambiente profundo de marisma de manglar, probablemente bajo constante inundación y seguido de condiciones más terrestres. Esto último, inferido por el aumento de polen de cultivos (Zea mays), y la disminución del polen de Ryzhopora (manglar rojo) (Kennett et al., 2004), la reducción de diatomeas marinas y la disminución en los indicadores de salinidad. Aquí se propone una laguna marginal o un ambiente de marisma somera para los ca. 2.300-2.500
A. P. (Ramírez-Herrera et al., 2007; RamírezHerrera et al., 2009). La explicación razonable de dicho cambio a un ambiente menos salino y menos inundado, es un probable desplazamiento vertical de la superficie (por levantamiento tectónico). Un descenso relativo del nivel del mar (se desconoce para el Holoceno en la costa Pacífica de México) o emergencia de la costa (levantamiento tectónico), ha sido propuesto para la laguna Tetitlán, al noroeste de la laguna Mitla, en ca. 2.270 A. P. (González-Quintero, 1980). Sin embargo, el registro sedimentario de los núcleos no permite discernir si se trata de un cambio repentino o gradual. Por lo que otra explicación alternativa podría ser la colmatación de la laguna. Las condiciones actuales de marisma de manglar, con una influencia marina corta en tiempo, se establece para los ca. 1.150 A. P.

Los cambios de nivel del mar ocurridos después de 6.000-5.200 A. P., que se observan en el registro estratigráficosedimentológico de laguna Mitla, probablemente obedecen a cambios inducidos por movimientos tectónicos ocasionados por el desplazamiento vertical de la costa (por ejemplo subsidencia por el movimiento de un segmento de la megafalla de la subducción mexicana), más que deberse a cambios absolutos en el nivel del mar o a cambios climáticos. Aunque el registro de cambio climático del Holoceno en México es relativamente limitado (Metcalfe et al., 2000), la información del centro y norte de México indica que las condiciones desérticas fueron establecidas para 4.000 A. P. (Metcalfe et al., 2000) en el norte de México, mientras que las montañas del centro registran un episodio seco que culminó en el 4.000 A. P., y un número de intervalos secos en los últimos 3.000 A. P. (Metcalfe et al., 2000). Un estudio local en una laguna costera de Sonora, al norte de México, sugiere que el clima era más húmedo en el $6.600 \mathrm{~A}$. P. (Caballero et al., 2005). Debido a las dificultades en correlacionar las variaciones del nivel del mar a nivel mundial, no ha sido posible establecer una curva universal del nivel del mar durante el Holoceno (Pirazolli, 1991). Se desconoce, hasta donde se sabe, de un óptimo, transgresión marina, en el Holoceno en la costa Pacífica mexicana, y no es congruente extrapolar datos de otras regiones, como los del Mar del 
Norte (Behre, 2003), a la costa de México. La información del clima en el Holoceno señala que los cambios estratigráficos, de microfósiles y geoquímicos que se observan en la laguna Mitla, no parecen ser el producto de cambios mayores en el clima y de transgresiones marinas. Por tanto, el aumento en la salinidad observado ca. 3.400-3.500 A. P. (Ramírez-Herrera et al., 2007; RamírezHerrera et al., 2009), no parece atribuirse a un cambio climático, y la subsidencia e incursión marina propuestos en este estudio es una explicación razonable para cambios de nivel de la costa por movimientos tectónicos e incursiones marinas. No es posible precisar la magnitud de la subsidencia cosísmica a partir de este registro geológico, sin embargo, se estima que debió ser del rango $>1$ $\mathrm{m}$ para cambiar el ambiente de la laguna a otros más salinos.

Basándose en la información científica que aquí se presenta, se puede subrayar las siguientes evidencias de subsidencia y una incursión marina (tsunami) en ca. 3.4003.500 A. P.: 1) el contacto abrupto basal entre unidades sedimentarias; 2) las capas de arena con clastos rasgados típico de depósitos de tsunami; 3) gradación normal, más finos hacia arriba; 4) laminaciones internas de los lodos; 5) el incremento repentino de la salinidad de la laguna registrado en los datos geoquímicos y de microfósiles, y acordes con, pero no exclusivos de una incursión de aguas marinas; y 4) el restablecimiento de las condiciones de salinidad reducida en los sedimentos en la parte superior de los núcleos estudiados, que apoyan la interpretación de que la salinidad alta no es una norma para la laguna Mitla. Nuevos elementos de análisis geoquímicos de los sedimentos, $\mathrm{S}$ y $\mathrm{Ba}$, corroboran cambios en la salinidad de los sedimentos atribuibles a una inundación marina y subsidencia en ca. 3.400-3.500 A. P. en la laguna Mitla.

En este artículo se refuerza con evidencias la propuesta de que los depósitos de arena aquí discutidos presentan características análogas a los depósitos de tsunami históricos y prehistóricos descritos en otros sitios del mundo (Kortekaas \& Dawson, 2007; Morton et al., 2007; Nichol et al., 2007; Jankaew et al., 2008; Monecke et al., 2008; Sawai et al., 2008).
Finalmente, se demuestra con nuevas evidencias que los cambios repentinos en la salinidad de la laguna no son atribuibles a cambios climáticos, ni de nivel del mar, ni a la rotura de la barra por tormentas excepcionales. Incorporando información de datos históricos se prueba que los sismos grandes en la zona de subducción mexicana son con frecuencia acompañados por deformación tectónica de la costa (subsidencia y/o levantamiento) y tsunamis, y que las evidencias presentadas de subsidencia cosísmica e inundación marina (tsunami) en la costa de Guerrero son razonables. Para discernir sobre la probabilidad de que la zona de subducción mexicana sea capaz de generar grandes terremotos, se enfatiza en la necesidad de realizar más estudios de paleo-sismicidad para conocer la extensión de estos depósitos de tsunami y de las evidencias de subsidencia cosísmica de este evento.

\section{Agradecimientos}

Gracias a Roger Byrne por las conversaciones y sugerencias relacionadas a este trabajo. Gracias a Liam Reidy por la lectura de la versión inicial de este manuscrito. Especial agradecimiento a José Antonio Navarrete por la ayuda en la elaboración de las figuras. Los comentarios de Juan A. Morales y F. Audemard M. mejoraron substancialmente este artículo. Se agradecen los comentarios de dos evaluadores anónimos que contribuyeron a mejorar este trabajo.

\section{Referencias bibliográficas}

AMBRASEYS, N. N.; JACKSON, J. A. \& MELVILLE, C. P. Historical seismicity and tectonics: the case of the Eastern Mediterranean and the Middle East: IASPEI. International Handbook of Earthquake and Engineering Seismology, 2002, № 81A, p. 747-763.

AMBRASEYS, N. N.; MELVILLE, C. P. \& ADAMS, R. D. The seismicity of Egypt, Arabia and the Red Sea. Cambridge: Cambridge University Press, 1994.

ANDERSON, R. S. \& MENKING, K. M. The Quaternary marine terraces of Santa Cruz, California: evidence for coseismic uplift 
on two faults. Geological Society of America Bulletin, 1994, № 106, p. 649-664.

ATWATER, B. F. Evidence for great Holocene earthquakes along the Outer coast of Washington State. Science, 1987, vol. 236, $\mathrm{N}^{\circ} 4804$, p. 942-944.

ATWATER, B. F. \& HEMPHILL-HALEY, E. Recurrence intervals for great earthquakes of the past 3500 years at northeastern Willapa Bay. Washington: U. S. Geological Survey, Professional Paper 1576, 1997.

ATWATER, B. F. \& MOORE, A. L. Evidence for great Holocene earthquakes along the outer coast of Washington State. Science, $1992, N^{\circ} 236$, p. $942-944$.

ATWATER, B. F.; FURUKAWA, R.; HEMPHILL-HALEY, E.; IKEDA, Y. \& KASHIMA, K. Seventeenth-century uplift in Eastern Hokkaido, Japan. Holocene, 2004, № 14, p. 487-501.

ATWATER, B. F.; HEDEL, C. W. \& HELLEY, E. J. Late Quaternary depositional history, Holocene sea-level changes, and vertical crustal movement, Southern San Francisco Bay. California: U. S. Geological Survey, Professional Paper 1014, 1977.

ATWATER, B. F.; NELSON, A. R.; CLAGUE, J. J.; CARVER, G. A.; YAMAGUCHI, D. K.; BOBROWSKY, P. T.; BOURGEOIS, J.; DARIENZO, M. E.; GRANT, W. C.; HEMPHILLHALEY, E.; KELSEY, H. M.; JACOBY, G. C.; NISHENKO, S. P.; PALMER, S. P.; PETERSON, C. D. \& REINHART, M. A., Summary of coastal geologic evidence for past great earthquakes at the Cascadia subduction zone. Earthquake Spectra, 1995, № 11, p. 1-18.

AUDEMARD, M. F. A. Estudios paleosísmicos por trincheras en Venezuela: métodos, alcances, aplicaciones, limitaciones y perspectivas. Revista geográfica venezolana, 2005, vol. 44, No 1, p. 11-46.

BEHRE, K. E. Eine neue Meeresspiegelkurve für die südliche Nordsee Transgressionen und Regressionen in den letzten 10.000. Jahren, 2003, brand 28, p. 9-63.

BERRYMAN, K. R. Age, height, and deformation of Holocene marine terraces at Mahia
Peninsula, Hikurangi subduction margin, New Zealand. Tectonics, 1993a, vol. 12, No 6, p. 1347-1364.

BERRYMAN, K. R. Age, height, and deformation of Late Pleistocene marine terraces at Mahia Peninsula, Hikurangi subduction margin, New Zealand: Tectonics, 1993b, 12(6), 1365-1379.

BILEK, S. L. Using earthquake source durations along the Sumatra-Andaman subduction system to examine fault-zone variations. Bulletin of Seismological Society of America, 2007, vol. 97, No 1, p. S62-S70.

BODIN, P. \& KLINGER, T. Coastal uplift and mortality of intertidal organisms caused by the September 1985 Mexico earthquakes. Science, 1986, № 233, p. 1071-1073.

BRIGGS, R. \& MELTZNER, A. Surface uplift and subsidence during the giant Sumatran megathrust ruptures of 2004 and 2005. AGU 2005 fall meeting. Eos, Transactions, American Geophysical Union, 2005, vol. 86, $\mathrm{N}^{\circ}$ 52, p. T31C-01.

BYRNE, R.; INGRAM, B. L.; STARRATT, S.; MALAMUD-ROAM, F.; COLLINS, J. N. \& CONRAD, M. E. Carbon-Isotope, Diatom and Pollen evidence for late Holocene Salinity change in a brackish marsh in the San Francisco Estuary. Quaternary Research, 2001, № 55, p. 66-76.

CABALLERO, M.; PENALBA, M. C.; MARTÍNEZ, M.; ORTEGA-GUERRERO, B. \& VÁZQUEZ, L. A Holocene record from a former coastal lagoon in Bahia Quino, Gulf of California, Mexico. The Holocene, 2005, № 15, p. 1236-1244.

CHAGÉ-GOFF, C. \& GOFF, J. R. Geochemical and sedimentological signature of catastrophic saltwater inundations (tsunamis), New Zealand. Quaternary Austraulasia, 1999, $N^{\circ} 17$, p. 38-48.

CHAGUÉ-GOFF, C.; DAWSON, S.; GOFF, J. R.; ZACHARIASEN, J.; BERRYMAN, K. R.; GARNETT, D. L.; WALDRON, H. M. \& MILDENHALL, D. C. A tsunami (c. 6.300 years BP) and other environmental changes, northern Hawke's Bay, New Zealand. Sedimentary Geology, 2002, No 150, p. 89-102. 
CHAPELL, J.; OTTA, Y. \& BERRYMAN, K. Late Quaternary coseismic uplift history of Huon Penincula, Papua New Guinea. Quaternary Science Reviews, 1996, № 15, p. 7-22.

CHINI, M. \& BIGNAMI, C. Uplift and subsidence due to the 26 December 2004 Indonesian earthquake detected by SAR data. International Journal of Remote Sensing, 2008, vol. 29, No 13-14, p. 3891-3910.

CISTERNAS, M. Suelos enterrados revelan la prehistoria sísmica del centro-sur de Chile durante los últimos dos milenios. Revista de Geografía Norte Grande, 2005, № 33, p. 19-31.

CISTERNAS, M.; ATWATER, B. F.; TORREJÓN, F.; SAWAI, Y.; MACHUCA, G.; LAGOS, M.; EIPERT, A.; YOULTON, C.; SALGADO, I.; KAMATAKI, T.; SHISHIKURA, M.; RAJENDRAN, C. P.; MALIK, J. K.; RIZAL, Y. \& HUSNI, M. Predecessors of the giant 1960 Chile earthquake. Nature, 2005, $\mathrm{N}^{\circ} 437$, p. 404-407.

CLAGUE, J. J.; BOBROWSKY, P. T. \& HUTCHINSON, I. A review of geological records of large tsunamis at Vancouver Island, British Columbia. Quaternary Science Reviews, 2000, No 19, p. 849-863.

CONTRERAS-ESPINOSA, F. \& WARNER, B. G. Ecosystem characteristics and management considerations for coastal wetlands in México. Hidrobiología, 2004, No 511, p. 233-245.

CORONA-ESQUIVEL, R.; ORTEGA-GUTIÉRREZ, F.; MARTÍNEZ-REYES, J. y CENTENO-GARCÍA, E. Evidencias de levantamiento tectónico asociado con el sismo del 19 de septiembre de 1985, en la región de Caleta de Campos, Estado de Michoacán. Revista Instituto de Geología, 1988, vol. 7, № 1, p. 106-111.

CUMMING, J. L., Los terremotos de junio de 1932 en los estados de Colima y Jalisco. Universidad de México, 1933, vol. VI, No 31 32, p. 68-104.

CUNDY, A. B.; KORTEKAAS, S.; DEWEZ, T.; STEWART, I. S.; COLLINS, P. E. F.; CROUDACE, I. W.; MAROUKIAN, H.; PAPANASTASSIOU, D.; GAKI-PAPANASTASSIOU,
P.; PAVLOPOULOS, K. \& DAWSON, A. Coastal wetlands as recorders of earthquake subsidence in the Aegean: a case study of the 1894 Gulf of Atalanti earthquakes, central Greece. Marine Geology, 2000, № 170, p. 3-26.

CUNDY, A. B.; SPRAGUE, D.; HOPKINSON, L.; MAROUKIAN, H.; GAKI-PAPANASSTASSIOU, K.; PAPANASSTASSIOU, D. \& FROGLEY, M. R. Geochemical and stratigraphic indicators of Late Holocene coastal development in the Gythio area, southern Peloponnese, Greece. Marine Geology, 2006, No 230, p. 161-177.

CURRAY, J. R.; EMMEL, F. J. \& CRAMPTON, P. J. S. Holocene history of a strand plain lagoonal coast, Nayarit, Mexico. En: UNAM-UNESCO. Lagunas costeras, Un simposio. Memorias Simposio Internacional, Lagunas costeras. Ciudad de México: UNAMUNESCO, 1969, p. 63-100.

DEMETS, C., GORDON, R., ARGUS, D. \& STEIN, S., Effect of recent revisions to the geomagnetic time-scale on estimate of current plate motions, Geophysical Research Letters, 1994, 21, 2191-2194.

FARRERAS, S. Tsunamis en México. En: LAVÍN, M. F. Contribuciones a la Oceanografía Física en México. Ciudad de México: Unión Geofísica Mexicana, Monografía № 3, 1997, p. 73-96.

FARRERAS, S. \& SÁNCHEZ, A. J. The tsunami threat on the Mexican west coast: A historical analysis and recommendations for hazard mitigation. Natural Hazards, 1991, No 4, p. 301-316.

FILINOV, A. E.; TERESHCHENKO, I. E. \& MONZON, C. O. Hydrographic monitoring of El Niño 97-98 off the coast of southwest Mexico. Geofísica Internacional, 2003, vol. 42, No 3, p. 307-312.

GERVEMEYER, I. \& TIWARI, V. M. Overriding plate controls spatial distribution of megathrust earthquakes in the Sunda-Andaman subduction zone. Earth and Planetary Science Letters, 2006, No 251, p. 199-208.

GONZÁLEZ-QUINTERO, L. Paleoecología de un sector costero de Guerrero, México 
(3.000 años). Memorias Coloquio sobre paleo-botánica y palinología., Colección Científica Prehistoria, 1980, № 86, p. 133-157.

GONZÁLEZ VILLASEÑOR, L. I. A preliminary study of the coloured organic acid content of the waters of coastal lagoons of Guerrero, Mexico. Anales del Centro de Ciencias del Mar y Limnologia, 1977, № 4, p. 243-247.

GUZMÁN-ARROYO, M.; MAÑON, O. S. y ORTIZ, P. M. A. Afinidad limnológica del sistema lagunar costero del estado de Guerrero, México. Boletín del Instituto de Geografía, 1986, № 16, p. 61-76.

HENSTOCK, T.; MCNEILL, L. C. \& TAPPIN, D. R. Seafloor morphology of the Sumatran subduction zone: Surface rupture during megathrusth earthquakes. Geology, 2006, vol. $34, N^{\circ} 6$, p. 485-488.

HERNÁNDEZ UNZÓN, A. Resumen de la temporada de ciclones tropicales 2007. Ciudad de México: Comisión Nacional del Agua y Secretaría de Medio Ambiente y Recursos Naturales. 2008a. Disponible en Internet: http://smn.cna.gob.mx/ciclones/tempo2007/ crt2007.pdf

HERNÁNDEZ UNZÓN, A. Informe sobre el pronóstico de la temporada de ciclones 2008. Ciudad de México: Comisión Nacional del Agua y Secretaría de Medio Ambiente y Recursos Naturales, 2008b. Disponible en Internet: http://smn.cna.gob.mx/ciclones/tempo2008/cn2008.html

HORTON, B. P.; ZONG, Y.; HILLIER, C. \& ENGELHART S. Diatoms from Indonesian mangroves and their suitability as sea-level indicators for tropical environments. Marine Micropaleontology, 2007, № 63, p. 155-168.

HUTCHINSON, I.; CLAGUE, J. J. \& MATHEWES, R. W. Reconstructing the tsunami record on an emerging coast: a case study of Kanim Lake, Vancouver Island, British Columbia, Canada. Journal of Coastal Research, $1997, N^{\circ} 13$, p. 545-553.

ISLAM, M. S. \& TOOLEY, M. J. Holocene sea-level movements and coastal dynamics: records of the last 9000 years at Panigati near Khulna, Bangladesh. In: ATKINS, P. J. \& ALAM, M. S. (eds.). Information technology, environment and development in Bangladesh. Durham: England, 1996, p. 84-100.

JANKAEW, K.; ATWATER, B. F.; SAWAI, Y.; CHOOWONG, M.; CHAROENTITIRAT, T.; MARTÍN, M. E. \& PRENDERGAST, A. Medieval forewarning of the 2004 Indian Ocean tsunami in Thailand. Nature, 2008, № 455, p. 1228-1231.

KASPER-ZUBILLAGA, J. J.; CARRANZAEDWARDS, A. \& ROSALES-HOZ, L. Petrography and geochemistry of Holocene sands in the western Gulf of Mexico: Implications for provenance and tectonic setting. Journal of Sedimentary Research, 1999, № 69, p. 10031010.

KAWANA, T. O. \& PIRAZZOLI, P. A. Holocene coastline changes and seismic uplift in Okinawa Island, the Ryukyus, Japan. Annals of Geomorphology, 1985, №57, p. 11-31.

KELSEY, H. M.; NELSON, A. R.; HEMPHILL-HALEY, E. \& WITTER, R. C. Tsunami history of an Oregon coastal lake reveals $4600 \mathrm{yr}$ record of great earthquakes on the Cascadia subduction zone. Geological Society of America Bulletin, 2005, vol. 117, N ${ }^{\circ}$ 7-8, p. 1009-1032.

KENNETT, D. J.; VOORHIES, B.; IRIARTE, J.; JONES, J. G.; PIPERNO, D.; RAMÍREZHERRERA, M. T. y WAKE, T. A. Avances en el proyecto Arcaico-Formativo: Costa de Guerrero. Ciudad de México: Instituto Nacional de Antropología e Historia, 2004.

KORTEKAAS, S. \& DAWSON, A. G. Distinguishing tsunami and storm deposits: an example from Martinhal, SW Portugal. Sedimentary Geology, 2007, № 200, p. 208-221.

KOSTOGLODOV, V. \& PONCE, L. Relationship between subduction and seismicity in the Mexican part of the Middle America trench. J. Geophysical Research, 1994, No 99, p. 729-742.

LOMNITZ, C. Major earthquakes and tsunamis in Chile during the period 1535 to 1955. Geological Rundschau, 1970, №59, p. 128-136. 
LONG, A. J. \& SHENNAN, I., Sea-Level Changes in Washington and Oregon and the 'Earthquake Deformation Cycle. Journal of Coastal Research, 1994, No 10, p. 825-838.

LÓPEZ-BUENDÍA, A. M.; BASTIDA, J.; QUEROL, X. \& WHATELEY, M. K. G. Geochemical data as indicators of paleosalinity in coastal organic-ruch sediments. Chemical Geology, 1999, № 157, p. 235-254.

MCCAFFREY, R. Understanding where a great earthquake can happen. In: Acapulco, American Geophysical Union Joint Assembly, 22-25 May 2007.

METCALFE, S. E.; O'HARA, S. L.; CABALLERO, M. \& DAVIES, S. J. Records of Late Pleistocene-Holocene climatic change in Mexico-a review. Quaternary Science Reviews, 2000, No 19, p. 699-721.

MICHETTI, A. M.; AUDEMARD, M. \& MARCO, S. Future trends in paleoseismology: Integrated study of the seismic landscape as a vital tool in seismic hazard analyses. Tectonophysics, 2005, vol. 408, No 1-4, p. 3-21.

MINOURA, K.; GUSIAKOV, V. G.; KURATOV, A.; TAKEUTI, S.; SVENDSEN, J. I.; BONDEVIK, S. \& ODA, T. Tsunami sedimentation associated with the 1923 Kamchatka earthquake. Sedimentary Geology, 1996, № 106 , p. $145-154$.

MINOURA, K.; NAKAYA, S. \& UCHIDA, $M$. Tsunami deposits in a lacustrine sequence of the Sanriku, Northeast Japan. Sedimentary Geology, 1994, No 89, p. 25-31.

MONECKE, K.; FINGER, W.; KLARER, D.; KONGKO, W.; MCADOO, B. G.; MOORE, A. L. \& SUDRAJAT, S. U. A 1,000-year sediment record of tsunami recurrence in northern Sumatra. Nature, 2008, No 455, p. 1232-1234.

MORTON, R. A.; GELFENBAUM, G. \& JAFFE, B. E. Physical criteria for distinguishing sandy tsunami and storm deposits using modern examples. Sedimentary Geology, 2007, No 200, p. 184-207.

NANAYAMA, F.; SATAKE, K.; FURUKAWA, R.; SHIMOKAWA, K. \& ATWATER, B. F. Unusually large earthquakes inferred from tsunami deposits along the Kuril trench. Nature, 2003, No 424, p. 660663.

NATAWIDJAJA, D. H.; SIEH, K.; CHLIEH, M.; GALETZKA, J.; SUWARGADI, B. W.; CHENG, H.; EDWARDS, R. L.; AVOUAC, J. P. \& WARD, S. N. Source parameters of the great Sumatran megathrust earthquake of 1797 and 1833 inferred from coral microatolls. Journal of Geophysical Research, 2006, No 111, p. B06403.

NELSON, A. R. \& PERSONIOUS, S. F. The potential for great earthquakes in Oregon and Washington: An overview of recent coastal geologic studies and their bearing on segmentation of Holocene ruptures, Central Cascadia subduction zone. In: ROGERS, A. M.; WALSH, T. J.; KOCKEMAN, W. J. \& PRIEST, G. R. (eds.). Assessing Earthquake Hazards and Redusing Risk in the Pacific Northwest. Washington: U. S. Geological Survey, Professional Paper 1560, 1996, p. 91-114.

NELSON, A. R.; SHENNAN, I. \& LONG, A. J. Identifying coseismic subsidence in tidal-wetland Stratigraphical sequences at the Cascadia subduction zone of western North America. Journal of Geophysical Research, 1996, No 101, B3, p. 6115-6135.

NI, S.; KANAMORI, H. \& HELMBERGER, D. Energy radiation from the Sumatra earthquake. Nature, 2005, No 434, p. 582.

NICHOL, S. L.; GOFF, J. R.; DEVOY, R. J. N.; CHAGUÉ-GOFF, C.; HAYWARD, B. \& JÁMESE, I. Lagoon subsidence and tsunami on the West Coast of New Zealand. Sedimentary Geology, 2007, No 200, p. 248-262.

NISHENKO, S. P. \& SINGH, S. K. Conditional probabilities for the recurrence of large and great interplate earthquakes along the Mexican subduction zone. Bulletin of the Seismological Society of America, 1987, $N^{\circ}$ 77, p. 2095-2114.

NÚÑEZ-CORNÚ, F. J.; ORTIZ, M. \& SÁNCHEZ, J. J. The great 1787 Mexican tsunami. Natural Hazards, 2008, vol. 47, No 3, p. 569-579. Disponible en Internet: http://www.springerlink.com/ content/52132479530n5425/ 
ORTIZ, M.; SINGH, S. K.; KOSTOGLODOV, V. \& PACHECO, J. Source areas of the Acapulco-San Marcos, Mexico earthquakes of 1962 (M 7.1; 7.0) and 1957 (M 7.7), as constrained by tsunami and uplift records. Geofisica Internacional, 2000, № 39, p. 337-348.

ORTLIEB, L. Nectonique et variations du niveau marin au Quaternaire dans la region du Golfe de Californie, Mexique. Doctorat d'Etat Thesis. Universite d'Aix-Marseille II, France, 1986.

OTTA, Y. \& CHAPPELL, J. Late Quaternary coseismic uplift events on the Huon Peninsula, Papua New Guinea, deduces from coral terrace data. Journal of Geophysical Research, 1996, vol. 101, No B3, p. 6071-6082.

PÁEZ-OSUNA, F. y OSUNA-LÓPEZ, J. I. Acumulación de metales pesados en Mitla, una laguna costera tropical. Ciencias Marinas, 1987, vol. 13, № 3, p. 97-112.

PARDO, M. \& SUÁREZ, G. Shape of the subducted Rivera and Cocos plates in southern Mexico: seismic and tectonic implications. Journal of Geophysical Research, 1995, vol. 100, No B7, p. 12357-12373.

PIRAZOLLI, P. A. World Atlas of Holocene sea level changes. Amsterdam: Elsevier, 1991.

RAMÍREZ-HERRERA, M. T. \& URRUTIAFUCUGAUCHI, J. Morphotectonic zones along the coast of the Pacific continental margin, southern Mexico. Geomorphology, 1999, $\mathrm{N}^{\circ} 28$, p. 237-250.

RAMÍREZ-HERRERA, M. T.; CUNDY, A. y KOSTOGLODOV, V. Probables sismos y tsunamis prehistóricos durante los últimos 5000 años en la costa de la brecha sísmica de Guerrero México, México. En: SOCIEDAD MEXICANA DE INGENIERÍA SÍSMICA. XV Congreso Nacional de Ingeniería Sísmica, 1-07. Ciudad de México: Sociedad Mexicana de Ingeniería Sísmica México D. F., 2005, p. 1-17.

RAMÍREZ-HERRERA, M. T.; CUNDY, A.; KOSTOGLODOV, V.; CARRANZA-EDWARDS, A.; MORALES, E. \& METCALFE, S. Sedimentary record of late-Holocene relative sealevel change and tectonic deformation from the Guerrero Seismic Gap, Mexican Pacific
Coast. The Holocene, 2007, vol. 17, № 8, p. 1211-1220.

RAMÍREZ-HERRERA, M. T.; CUNDY, A. B.; KOSTOGLODOV, V. \& ORTIZ, M. Late Holocene tectonic-land level changes and tsunamis at Mitla lagoon, Guerrero, Mexico. Geofísica Internacional, 2009, vol. 48, № 2, p. 195-209.

RAMÍREZ-HERRERA, M. T.; KOSTOGLODOV, V.; SUMMERFIELD, M. A.; URRUTIAFUCUGAUCHI, J. \& ZAMORANO, J. J. A reconnaissance study of the morphotectonics of the Mexican subduction zone. Annals of Geomorphology, 1999, № 118, p. 207-226.

REIMER, P. J.; BAILLIE, M. G. L.; BARD, E.; BAYLISS, A.; BECK, J. W.; BERTRAND, C. J. H.; BLACKWELL, P. G.; BUCK, C. E.; BURR, G. S.; CUTLER, K. B.; DAMON, P. E.; EDWARDS, R. L.; FAIRBANKS, R. G.; FRIEDRICH, M.; GUILDERSON, T. P.; HOGG, A. G.; HUGHEN, K. A.; KROMER, B.; MCCORMAC, F. G.; MANNING, S. W.; RAMSEY, C. B.; REIMER, R. W.; REMMELE, S.; SOUTHON, J. R.; STUIVER, M.; TALAMO, S.; TAYLOR, F. W.; VAN DER PLICHT, J. \& WEYHENMEYER, C. E. IntCal04 Terrestrial radiocarbon age calibration, 26 - 0 ka BP. Radiocarbon, 2004, № 46, p. 1029-1058.

RUFF, L. \& KANAMORI, H. Seismicty and the subduction proceses. Physics of the Earth and Planetary Interiors, 1980, № 23, p. 240252.

SATAKE, K. \& ATWATER, B. F. Long-term perspectives on giant earthquakes and tsunamis at subduction zones. Annual Review of Earth and Planetary Sciences, 2007, № 35, p. 349-374.

SATAKE, K.; SHIMAZAKI, K.; YOSHINOBU, T. \& UEDA, K. Time and size of a giant earthquake in Cascadia inferred from Japanese tsunami rcords of January 1700 . Nature, 1996, No 379, p. 246-249.

SAWAI, Y. Episodic emergence in the past 3000 years at the Akkeshi estuary, Hokkaido, northern Japan. Quaternary Research, 2001, $\mathrm{N}^{\circ} 56$, p. 231-241.

SAWAI, Y.; FUJII, Y.; FUJIWARA, O.; KAMATAKI, T.; KOMATSUBARA, J.; OKAMURA, 
Y.; SATAKE, K. \& SHISHIKURA M. Marine incursions of the past 1500 years and evidence of tsunamis at Suijin-numa, a coastal lake facing the Japan Trench. The Holocene, 2008, vol. 18, NN$^{\circ}$, p. 517-528.

SAWAI, Y.; SATAKE, K.; KAMATAKI, T.; NASU, H. \& SHISHIKURA, M. Transient uplift after a seventeenth-century earthquake along the Kuril subduction zone. Science, 2004, No 306, p. 1918-1920.

SECRETARÍA DE COMUNICACIONES Y TRANSPORTES (SCT). Condicionantes físicas: dirección general de puertos-S.C.T. Ciudad de México: SCT, 2008. Disponible en Internet: http://e-mar.sct.gob.mx/fileadmin/PNDP2008/ doc/mdi/dcap03.pdf

SHENNAN, I.; SCOTT, D. B.; RUTHERFORD, M. \& ZONG, Y. Microfossil analysis of sediments representing the 1964 earthquake, exposed at Girdwood Flats, Alaska, USA. Quaternary International, 1999, № 60, p. 55-73.

SINGH, S. K. \& SUÁREZ, G. Review of the Seismicity of Mexico with Emphasis on the September 1985, Michoacan Earthquakes. Ciudad de México: Instituto de Geofísica, UNAM, 1986.

SINGH, S. K.; HAVSKOV, J. \& ASTIZ, L. Seismic gaps and recurrence periods of large earthquakes along the Mexican subduction zone. Bulletin of the Seismology Society of America, 1981, № 71, p. 827-843.

SIRKIN, L. Late Quaternary stratigraphy and environments of the west coastal plain. Palynology, 1985, № 9, p. 3-25.

SPENCER, K.; CUNDY, A. B. \& CROUDACE, I. W. Heavy metal distribution and earlydiagenesis in salt marsh sediments from the Medway estuary, Kent, U. K.. Estuarine, Coastal and Shelf Science, 2003, No 57, p. 43-54.

STEIN, S. \& OKAL, E. A. Speed and size of the Sumatra earthquake. Nature, 2005, No 434, p. 581-582.

STUIVER, M. \& REIMER, P. J. Extended ${ }^{14} \mathrm{C}$ database and revised CALIB radiocarbon calibration program. Radiocarbon, 1993, Nº 35 , p. 215-230.
SUÁREZ, G. How Large can Mexican Subduction Earthquakes be? Evidence of a Very Large Event in 1787 (M 8.5). In: Acapulco, American Geophysical Union Joint Assembly, 22-25 May 2007.

SUÁREZ, G.; MONTFRET, T.; WITTLINGER, G. \& DAVID, C. Geometry of subduction and depth of the seismogenic zone in the Guerrero Gap, Mexico. Nature, 1990, № 345, p. 336-338.

SUBARYA, C.; CHLIEH, M.; PRAWIRODIRDJO, L.; AVOUAC, J. P.; BOCK, Y.; SIEH, K.; MELTZNER, A. J.; NATAWIDJAJA, D. H. \& MCCAFFREY, R. Plate-boundary deformation associated with the great Sumatra-Andaman earthquake. Nature, 2006, No 440, p. 46-51.

TOBITA, M. \& SUITO, H. Outline of vertical displacement of the 2004 and 2005 Sumatra earthquakes revealed by satellite radar imagery. Earth, Planets and Space, 2006, vol. 58, No 12 , p. e1-e4.

UNAM SEISMOLOGY GROUP. The September 1985 Michoacan earthquakes: aftershock distribution and history of rupture. Geophysical Research Letters, 1986, No 13, p. 573-576.

VALDÉS-GONZÁLEZ, C. \& NOVELOCASANOVA, D. A. The western Guerrero, Mexico, seismogenic zone from the microseismicity associated to the 1979 Petatlan and 1985 Zihuatanejo earthquakes. Tectonophysics, 1998, № 287, p. 271-277.

VIRKANEN, J. The effects of natural environmental changes on sedimentation in Lake Kuttanen, a small closed lake in Finnish Lapland. The Holocene, 2000, № 10, p. 377-386.

VORREN, K. D. \& ALM, T. Late Weichselian and Holocene environments of lake Endletvatn, Andoya, northern Norway: as evidenced primarily by chemostratigraphic data. Boreas, 1999, № 28, p. 505-520.

WANG, P. \& HORWITZ, M. H. Erosional and depositional characteristics of regional overwash deposits caused by multiple hurricanes. Sedimentology $y_{\llcorner} 2006$, p. 1-20. 
WILLIAMS, H. \& HUTCHINSON, I. Stratigraphic and microfossil evidence for two late Holocene tsunamis at Swantown marsh, Whidbey Island, Washington State. Quaternary Research, 2000, No 54, p. 218-227.

ZONG, Y. \& TOOLEY, M. J. Holocene sealevel changes and crustal movement in Morecambe Bay, northwest England. Journal of Quaternary Science, 1996, № 11, p. 43-58.
ZWOLSMAN, J. J. G.; BERGER, G. W. \& VAN ECK, G. T. M. Sediment accumulation rates, historical input, postdepositional mobility and retention of major elements and trace metals in salt marsh sediments of the Scheldt estuary, SW Netherlands. Marine Chemistry, 1993, No 44, p. 73-94. 